\title{
Termologia del lago di Bracciano: onde interne ${ }^{(*)}$
}

\author{
PIETRO CALOI
}

1. - In precedenti lavori, sono stati portati aicuni contributi $\left({ }^{1},{ }^{2},{ }^{3}\right)$ alla conoscenza dei moti liberi e forzati del lago di Braceiano, preso nel suo insieme, considerato cioè come bacino oscillante nella sua interezza.

Si è provato come i valori trovati jer via teorica trovino ot timo accordo nelle osservazioni, sia per le oscillazioni libere simmetriclee clie por quelle asimmetriche.
Elaborando le registrazioni di oscillazioni libere, ottenute in diverse epoclie sul lago di Bracciano, avevo constatato l'esistenza, durante i mesi estivi da $A$ gosto ad Ottobre, di oscillazioni lunglissime, con un periodo approssimativo di sei ore.

Ho esegruito opportune riduzioni (nell'asse dei tempi) di alcuni tratti di registrazione, relative ai mesi estivi del 1956.

SESSE TEAMICHE

BRACCIANO

14. Agosto 1956

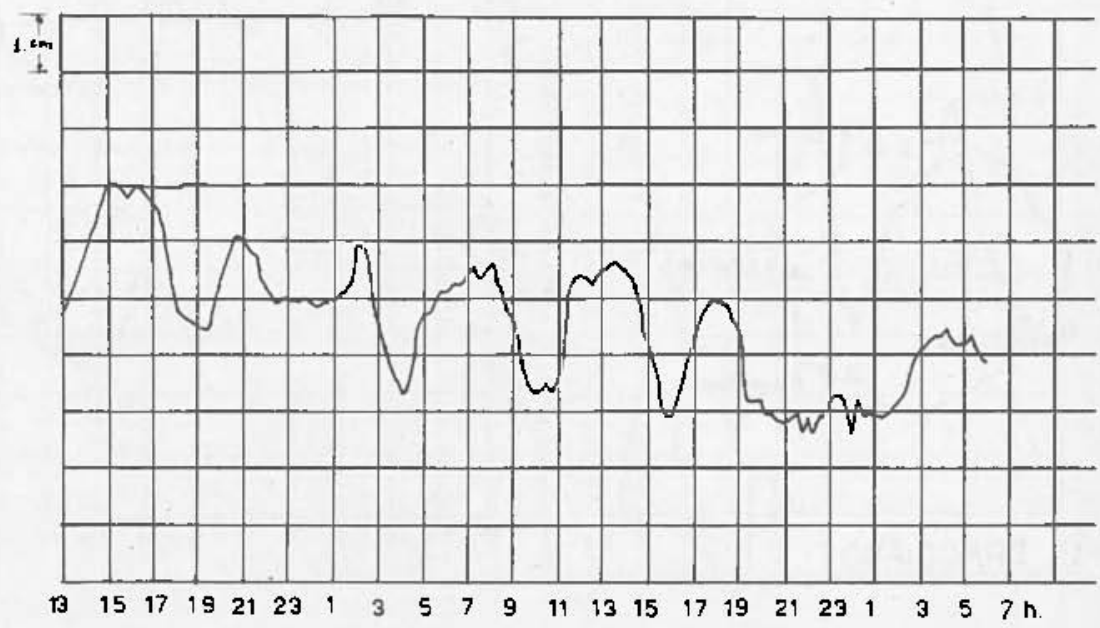

Fig. 1. - Rirluzione di registrazioni da limnografo non filtrato: chiari esempi di onde interne uninotali, con una binotlale e perturbazioni per sesse orlinarie.

Fia l'altro, è risultato che l'oscillazione liberı di massimo periodo è l'uninodale asimmetrica di $8 \mathrm{~m}, 3$.

Si è visto inoltue che, in condizioni barometriche particolasi, si possono fomare oscillazioni forzate di vario periodo, con valori the jossono raggiungere la mezz'ora, e di ampiezze talvolta notevoli, oscillazioni che però si smorzano e si annullano rapidamente.
Poichè i limnografi, destinati in quell'epoca alla registrazione delle onde libere del lago, come si è visto, di periodi relativamente jiccoli, erano poco tiltuati, le oscillazioni di lunghissimo periodo sono più o meno alterate da soviapposte oscillazioni a breve periodo. Ad ogni modo, ciò malgrado, dalla tig. 1, appare chiaro l'insorgere di ampie oscillazioni - sia pure molto deformate di un periodo medio di $6^{14}$. Nelia fig. 2, le

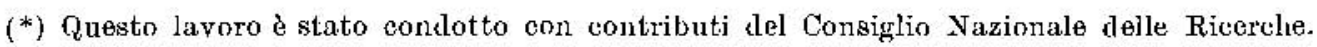


SESSE TERMICHE

BRACCIANO

15 Agosio 1956

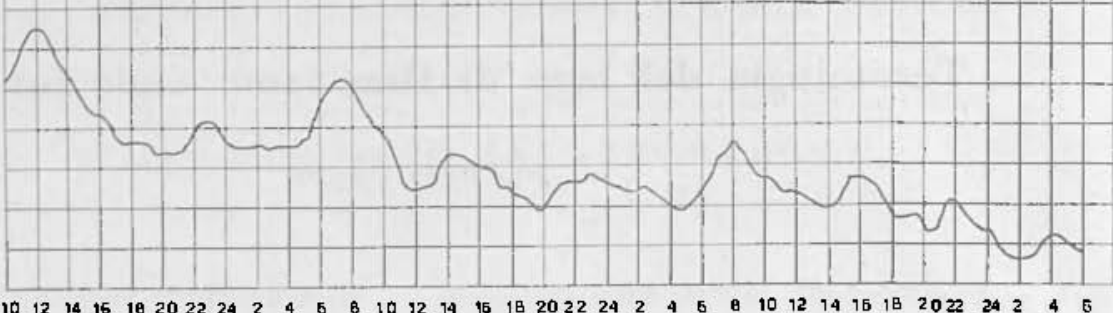

Fig. 2. - Nuovi esempi a Bracciano.

SESSE TERMICHE
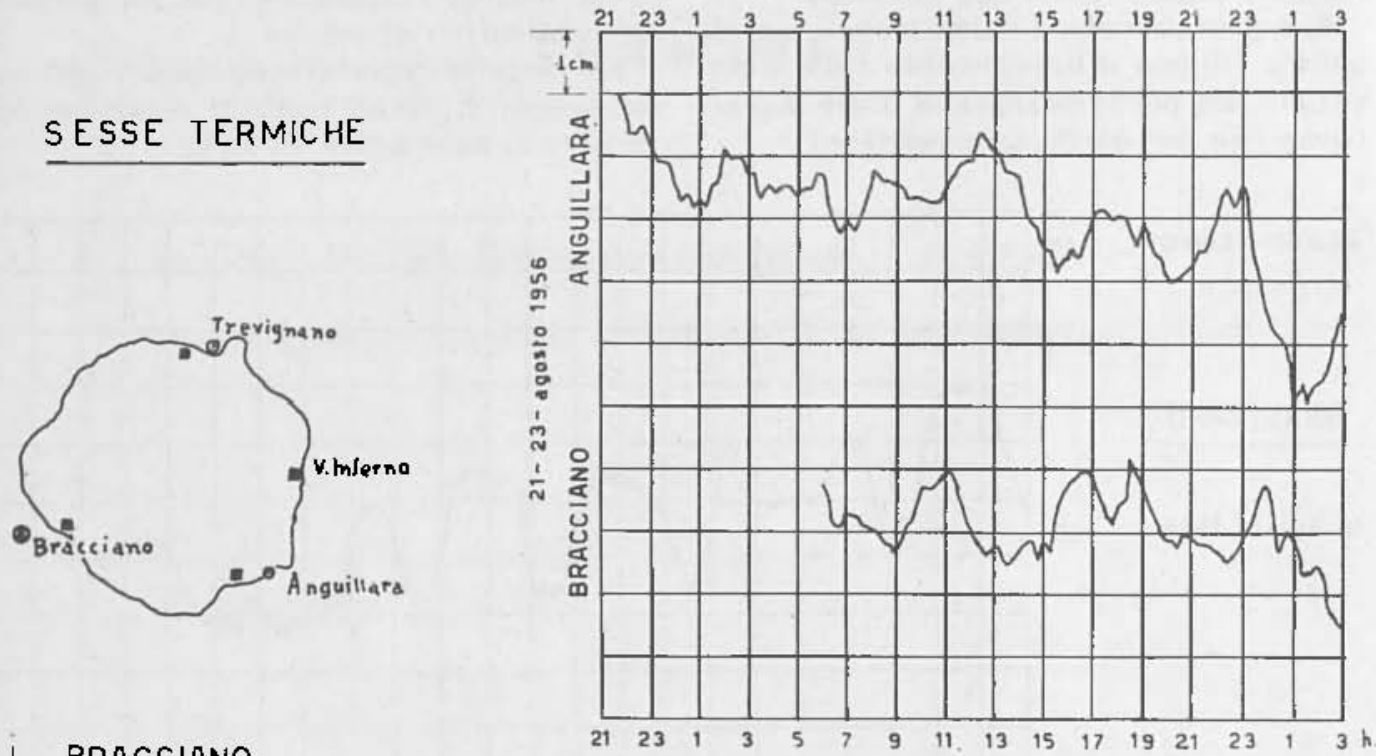

L. BRACCIANO

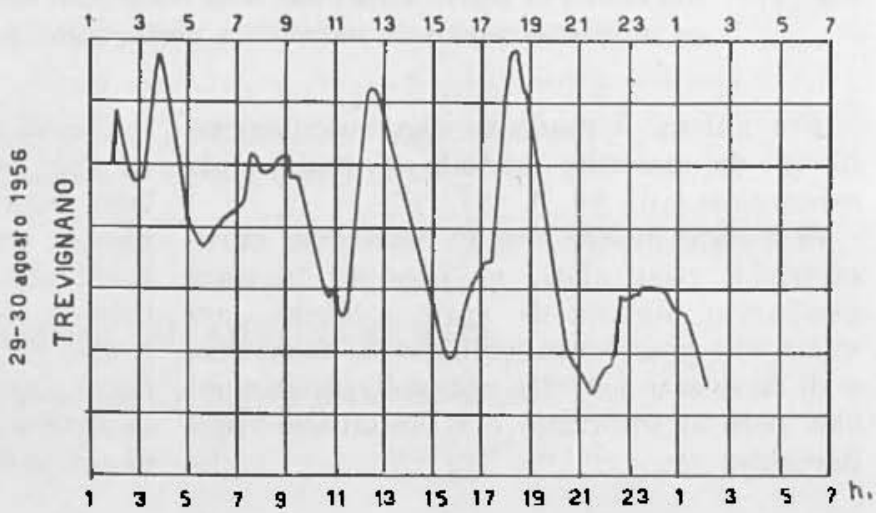

Fig. 3. - Riduzione da limnogrammi non filtrati: onde interne a Anguillara, Bracciano e Trevignano. 


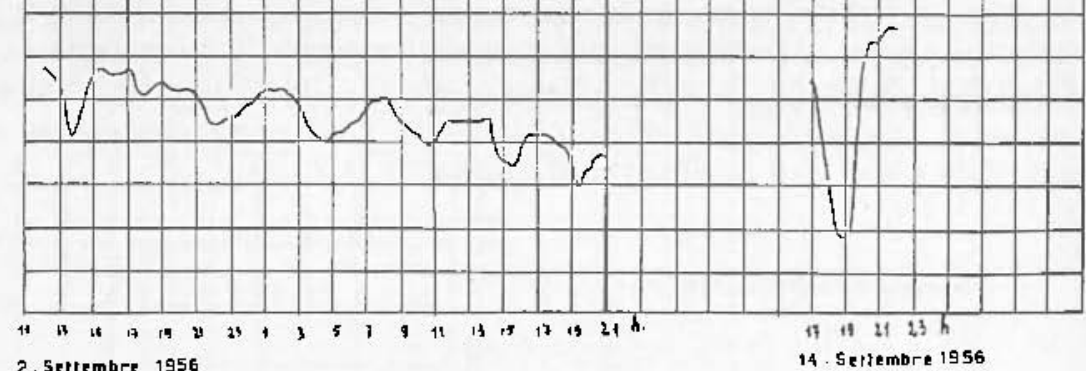

Fig. 4. - Chiari esempi onde interne uninodali a Trevignano.

\section{SESSE TERMICHE}
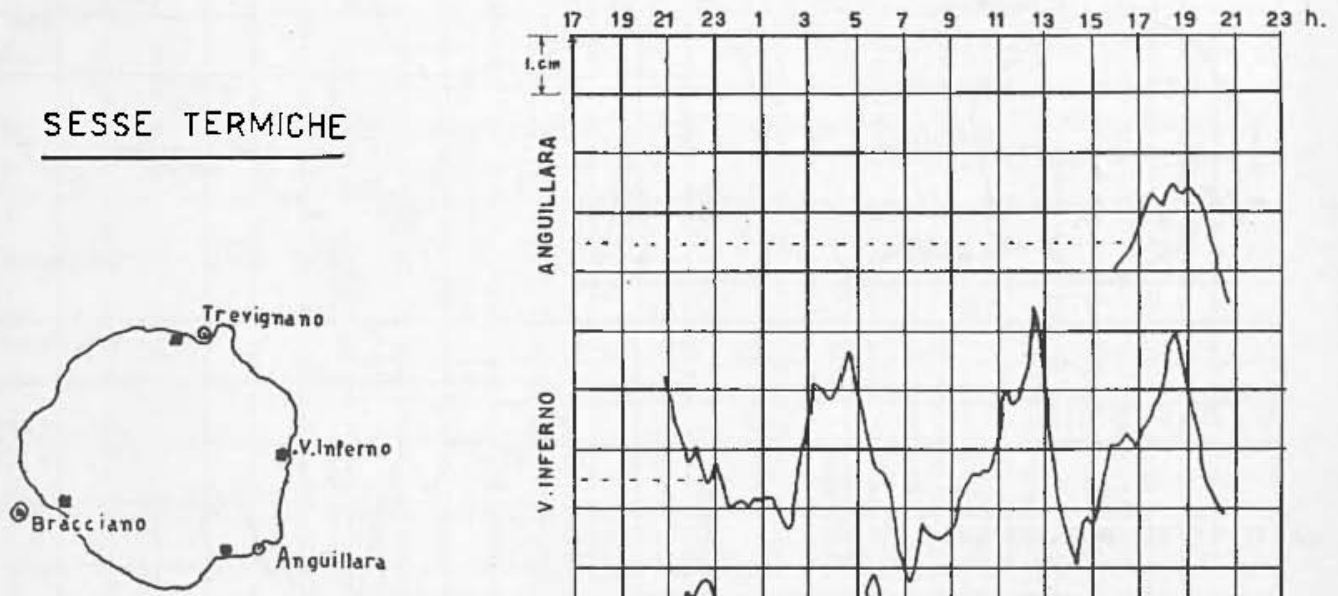

L. BRACCIANO

dal 14 SET TEMBRE 1956 h.17

al $15 \quad$.. $\quad$, h. 23
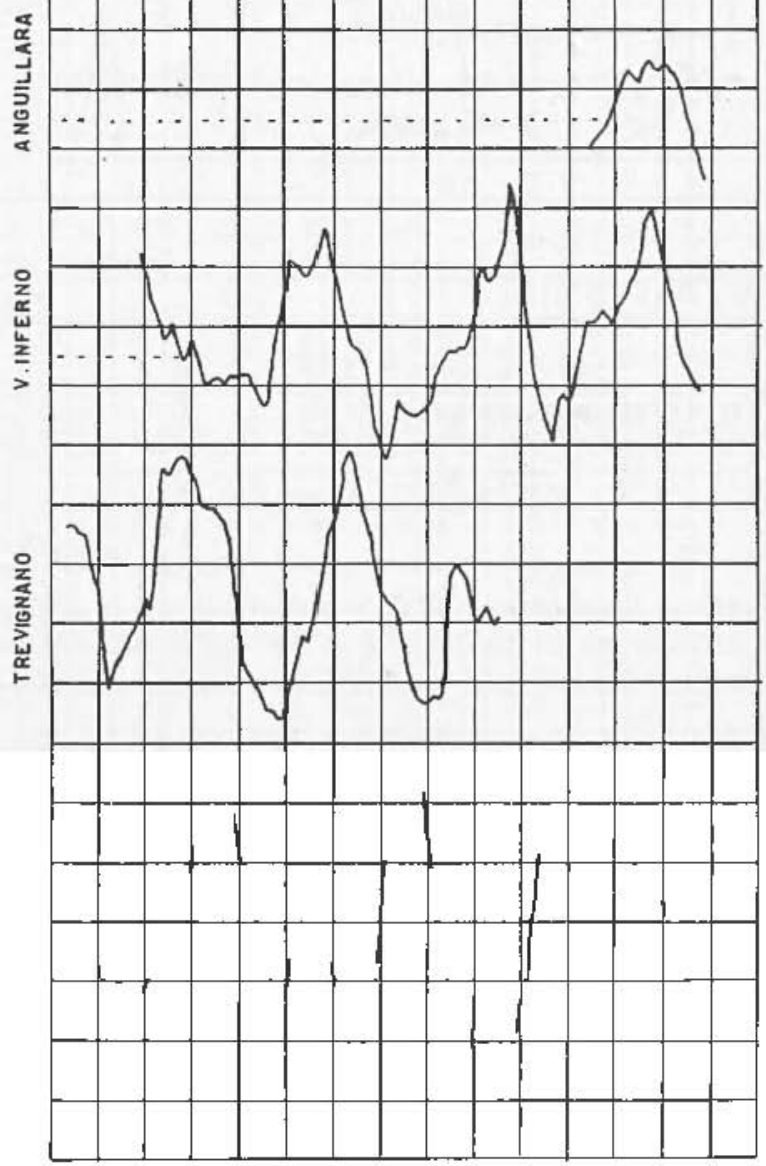

Figr. 5. - Onde interne nel lago di Bracciano, tratte dalla riduzione di registrazioni, in quattro stazioni, di limnograf per sesse ordinarie; particolarmente ampia l'onda interna uninodale, che si presenta in opposizione di fase a Bracciano e a Valle dell'Inferno. 
oscillazioni in parola appariscono di minor ampiezza c isolate, come se la causa perturbante agisse ad intervalli, più o meno distanziati. Nella fig. 3, a Bracciano e ad
Di particolare interesse sono le registrazioni ottenute il 14 e il 15 Settembre nelle stazioni limnografiche in funzione sul lago di Bracciano. Le oscillazioni con periodo

\section{SESSE TERMICHE}

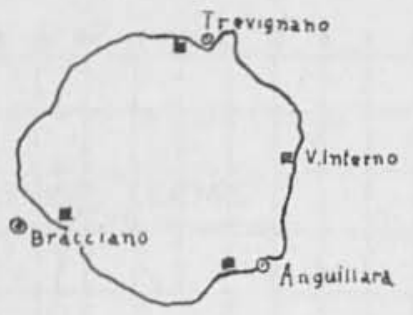

L. BRACCIANO

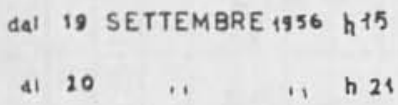

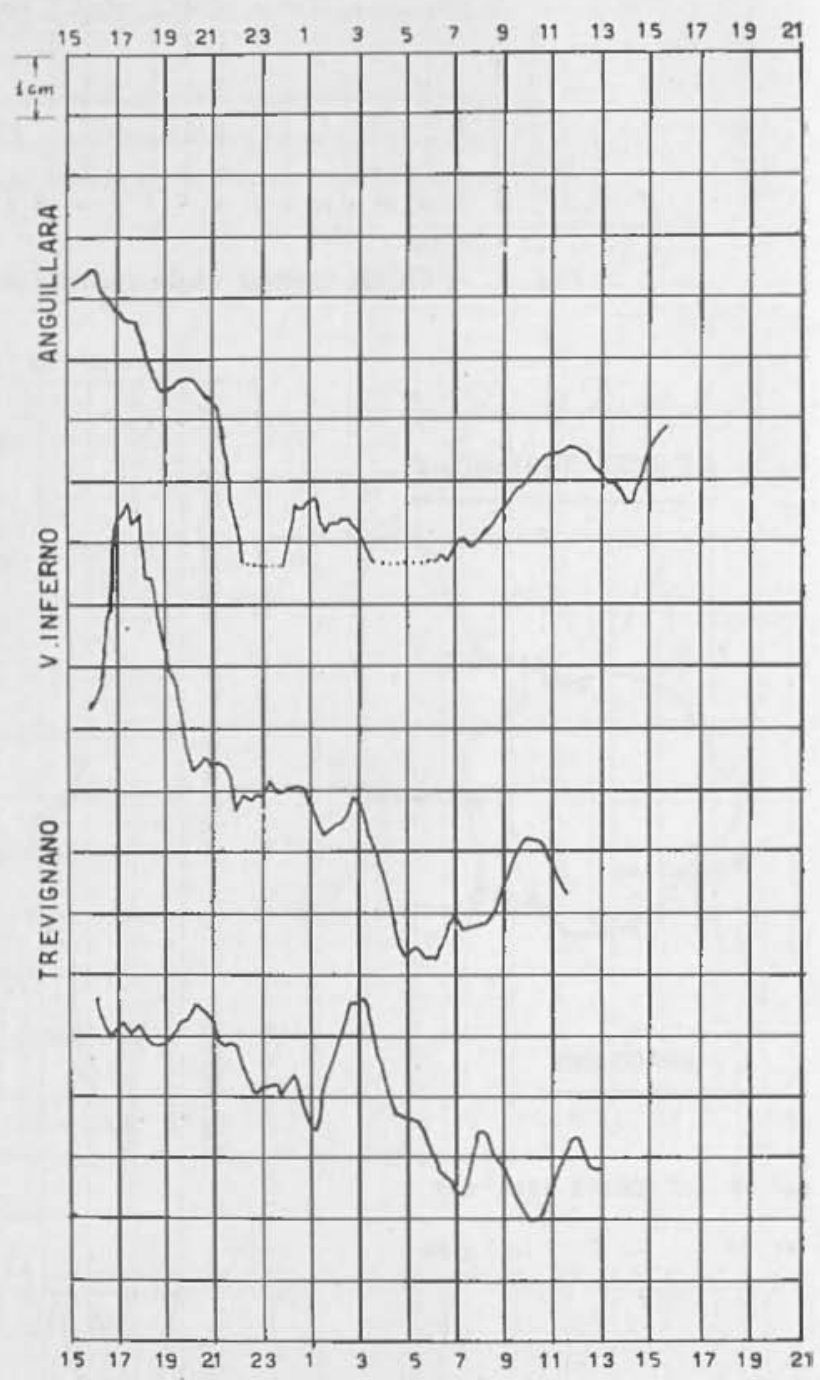

Fig. 6. - Esempi molto perturbati, con livello del lago in diminuzione.
Anguillara, appaiono perturbatissime e si presentano in opposizione di fase; nettissimo, nella stessa figura, l'esempio registrato a Trevignano dal 29 al 30 Agosto. Altri chiari esempi di oscillazioni con periodo dell'ordine di $6^{\text {h }}$ si ebbero a Trevignano il 2 Settembre 1956 e, amplissimo, il 14 Settembre (fig. 4). di $6^{\text {b }}$ circa appaiono ampie su tutto il lago: esse si presentano in fase a Valle dell'Inferno e ad Anguillara, che sono invece in opposizione di fase con Bracciano c, prevalentemente, anche con Trevignano. Si tratta indubbiamente di onde stazionarie uninodali $-o$, comunque, di nodalità dispari Nel caso specifico, insorgono a gruppi sepa- 
rati, un gruppo nascendo mentre il precedente è ancora in registrazione. Fra le oseillazioni sovrapposte, di diversa natura, figurano sicuramente anche delle armoniche, come quelle che si presentano verso le 5 del 14 Settembre.
2. - Il periodo delle oscillazioni osservate - cosi discosto diłl periodo dell'oscillazione libera fondamentale $(1,2)-$, indicava chiaramente trattarsi di oscillazioni di natura diversa da quelle libere ordinarie (sesse or(inarie). D'altronde, la loro persistenza, nei

\section{SESSE TERMICHE}
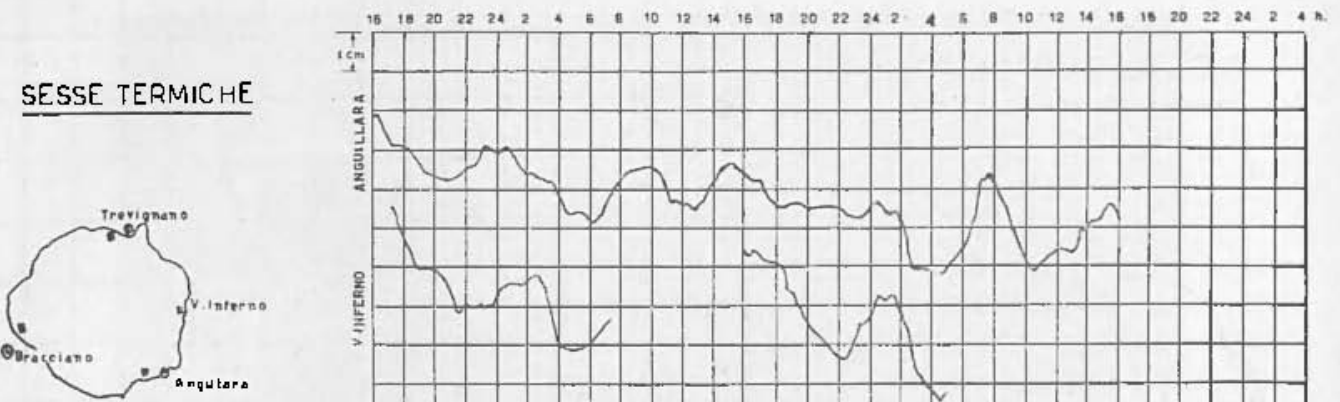

L. BRACCIANO

Lat 20 settembre 1950 in 15
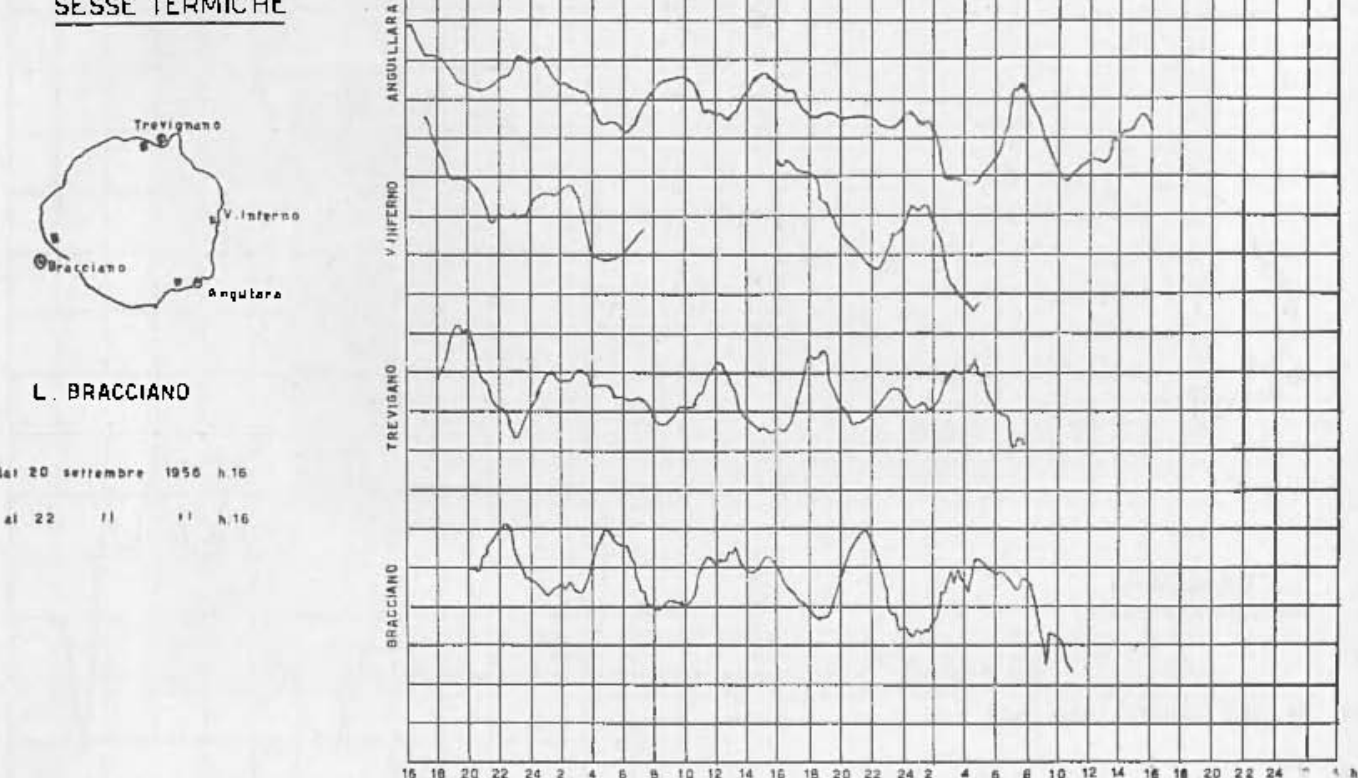

Fig. 7. - Onde interne nel lago di Braceiano, come da riduzione delle registrazioni ordinarie in quattro stazioni limnografiche. Le uninodali si presentano in opposizione di fase nelle eoppie di stazioni Braeciano-Valle dell'Inferno, Anguillara-Trevignano.

Sebbene sembri avere prevalentemente la direzione NE-SW, l'uninodo compie rotazioni intorno al centro del lago, quasi questi avesse le funzioni di un punto anfidromico; cio appare chiaro dalle figure precedenti, come dalla fig. 6 , e forse più ancora dalla fig. 7 .

La fig. 8 prova l'esistenza dj una varietà di altre oscillazioni che, pur non essendo del tipo ora esaminato, e nemmeno sesse ordinarie, tengono in ogni modo mosso il lago durante intere giornate. Non è escluso che parecchie di esse risultino essere partjcolari tipi di onde progressive. mesi della tarda estate e del primo autunno, permetteva di escludere di poterle identificare con oscillazioni forzate dell'intera massa del lago, provocate da cause accidentali $\left({ }^{2}\right)$.

Ritenni doversi trattare di onde interne (sesse termiche), sia pure di ampiezza e in sequenze eccezionali.

Non restava che la prova diretta.

Si doveva provare il formarsi - nei mesi estivi - autunnali - delle condizioni necessarje per l'insorgenza di tali oscillazioni. In altri termini, bisognava provare l'esistenza - in detti mesi - iello strato termico di salto e quindi di una superficie $d j$ 
discontinuità termica, ad una prodondità tale, e con tali caratteristiche, da giustificare l'insorgere delle osservate oscillazioni, di quel determinato periodo, sotto l'azione di cause perturbanti.

SESSE TERMICHE

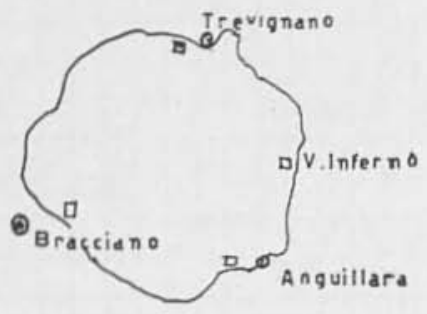

\section{BRACCIANO}

\section{dA I 24 SETTEMBRE $1956 \quad h .45$}

2i 25 I. h. 21

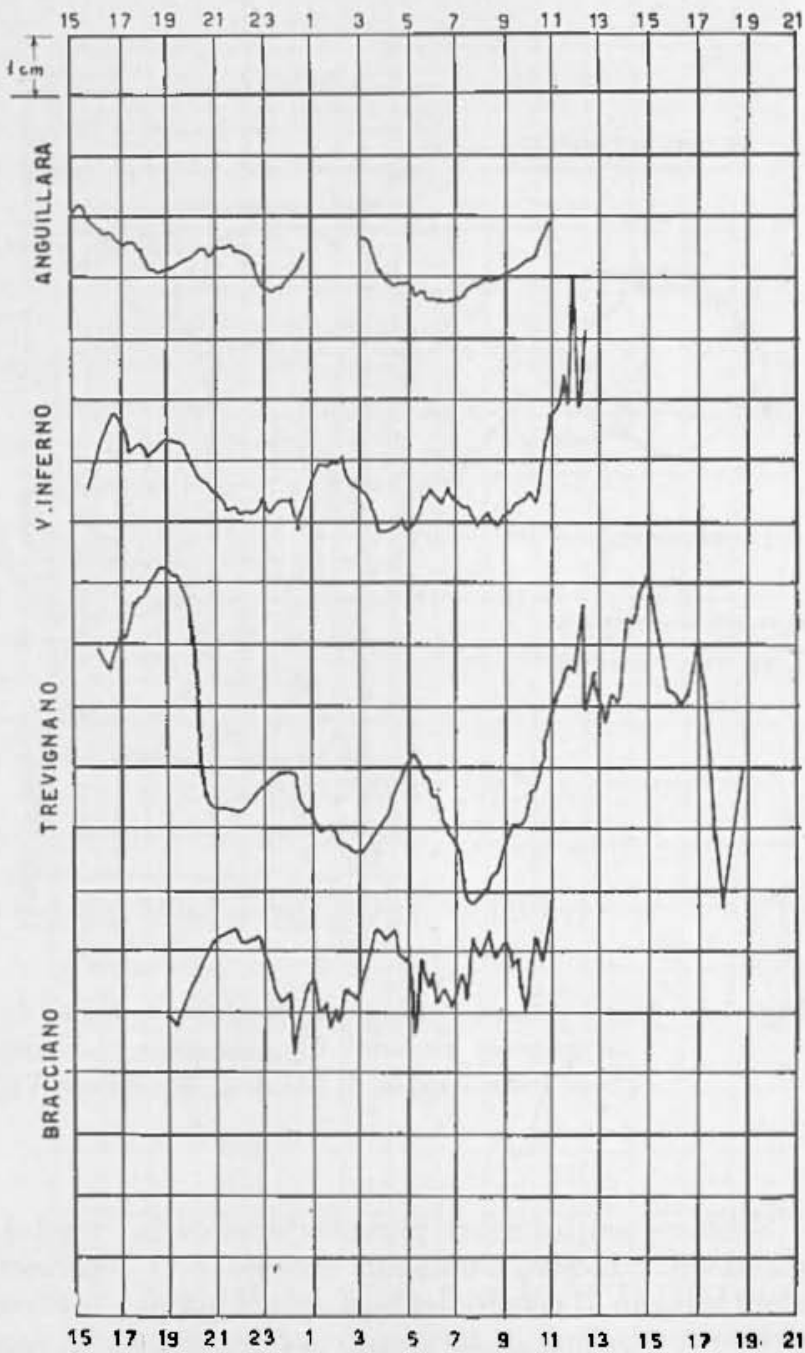

Fig. 8. - Registrazioni molto perturbate.

Necessitava pertanto una lunga serie di misure termometriche, nelle acque del lago, per profondità diverse $\mathrm{e}$ in diverse stagioni dell'anno.

I termometri a rovesciamento, che conducono ad osservazioni di grande precisione, avrebbero richiesto permanenze troppo lun- glie sul lago, per il conseguimento dello scopo.

Almeno in un primo tempo, ho preferito ricorrere ad uno strumento, forse meno preciso, ma in compenso di uso più agevole $\mathrm{e}$ 
Bourdon per profondità fino a 300 metri, con contemporanea registrazione della temperatura mediante una Ismina bimetallica.

L'apparecchio può essere ancorato stabilmente, oppure incorporato in un involucro di protezione, trascinabile. a tempo, incorporato nell'orologeria (12). In tal modo, mediante la staffia battente, vengono premuti i martelli prismatici del tubo Bourdon e della lamina bimetallica sul listello d'arresto, incorporato nell'apparechio registratore. Sulla carta cerata,

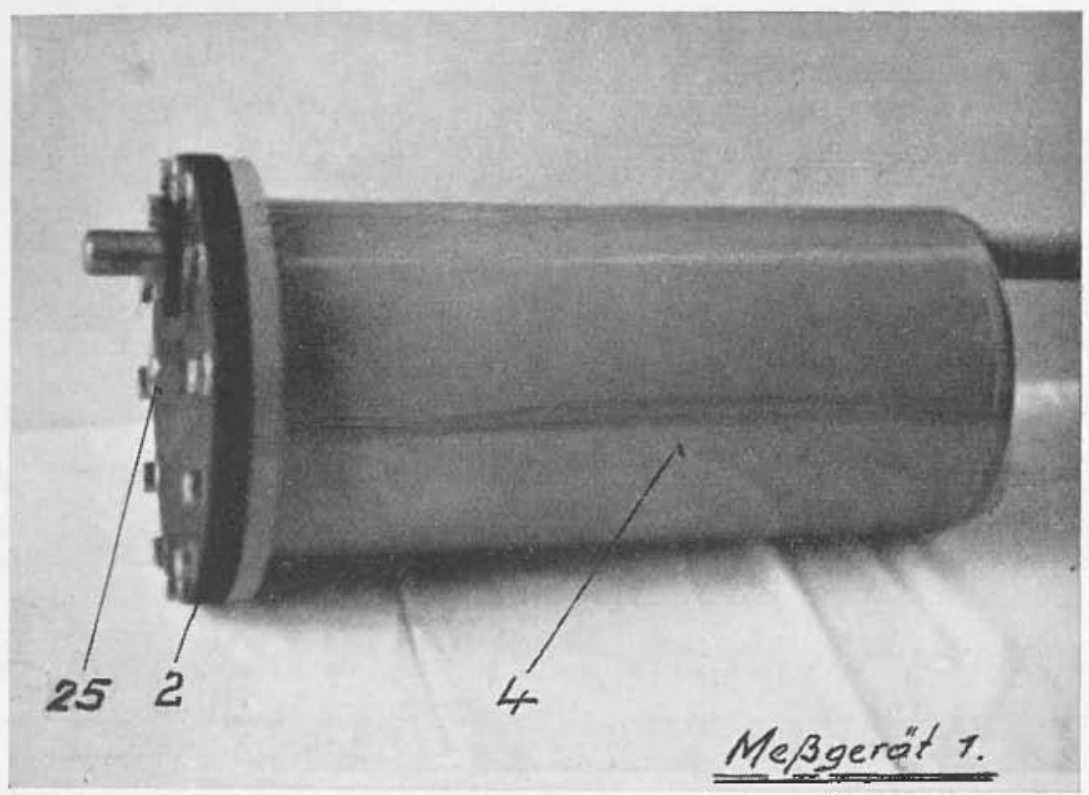

Fig. 9.

Si compone (figg. 9, 10, 11) di un involucro cilindrico del diametro di em 25, lungo cm 50 (4), di un coperohio (2) e di un basamento (3).

Il tubo Bourdon viene applicato al coperchio ed ̀̀ intercambiabile per determinate profondità.

Sull'asse di misura del dispositivo di trasmissione del tubo Bourdon ̀̀ applicato, mediante una vite di bloceaggio, l'indice con martelletto prismatico. Pure al coperchio è applicata la lamina bimetallica con mastello prismatico.

Il magnete battente (10) è sistemato al disopra del tubo Bourdon: a distanza rispettivamente di 1 minuto oppure di 12 secondi, esso riceve - una volta avviato il registratore (15) - un impulso di corrente che viene da pile a secco, collegate in serie, mediante contatti di un dispositivo che trovasi fra martello e listello d'arresto, si segna così un punto.

L'involucro di protezione trascinabile ha dimatero di cm 50 e lunghezza di m 1 . Il supporto cardanico dell'apparecchio di misura permette una sua posizione orizzontale, anche se l'involucro di protezione gira intorno al suo asse longitudinale.

Per quanto riguarda il rilevamento delle pressioni, a seconda delle profondità del lago in esame e degli scopi che si perseguono, sono a disposizione tre tubi Bourdon, con campi di misura di 300 metri, 100 metri e 20 metri rispettivamente.

La precisione assoluta d̀ di circa $0,3 \%$ del relativo campo di misury del tubo Bourdon. La precisione relativa è maggiore, in modo che i valori di variazione di pressione d'acqua misurati, possono ritenersi praticamente esatti. 


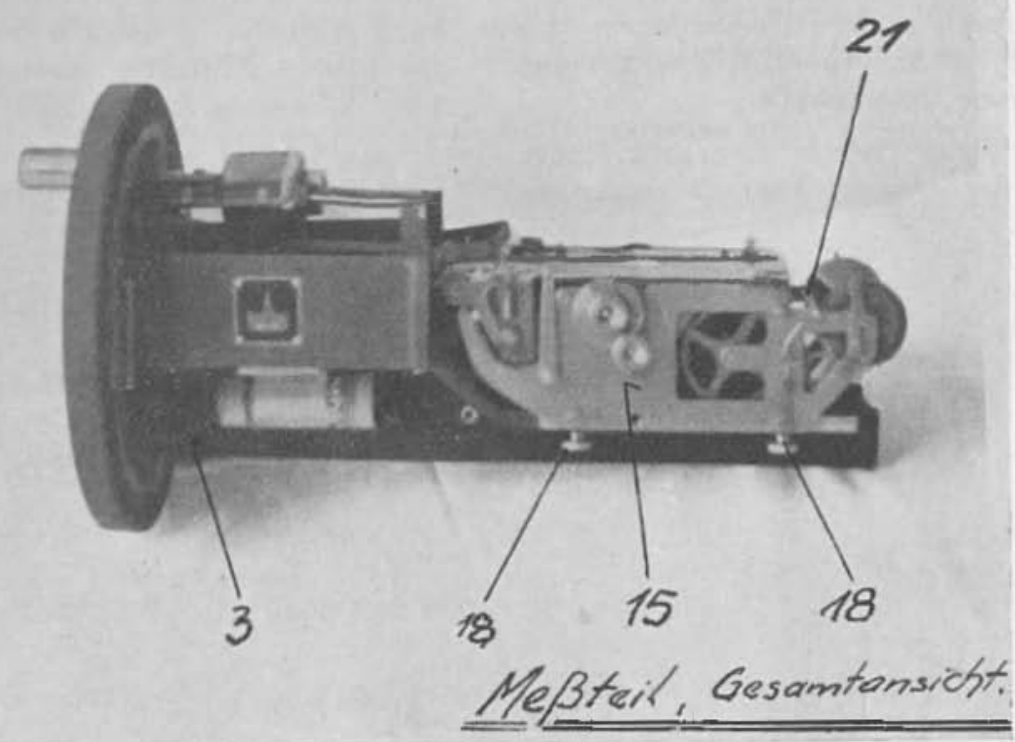

Fig. 10.

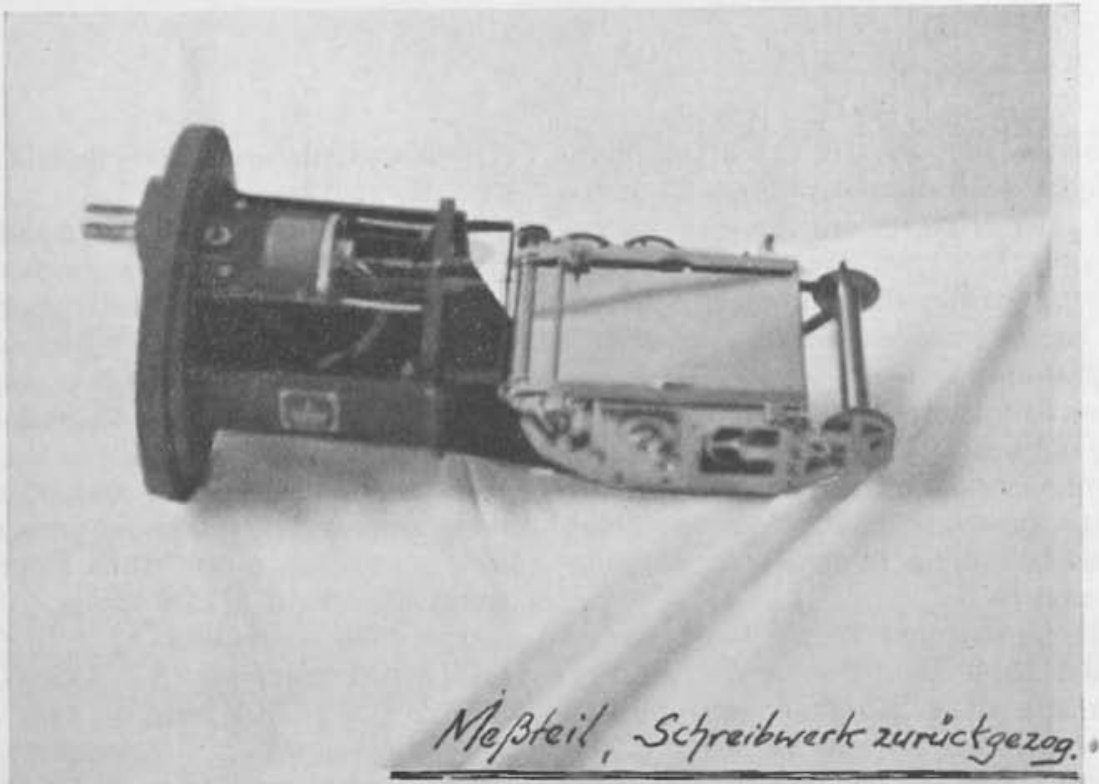

Fig. 11. 


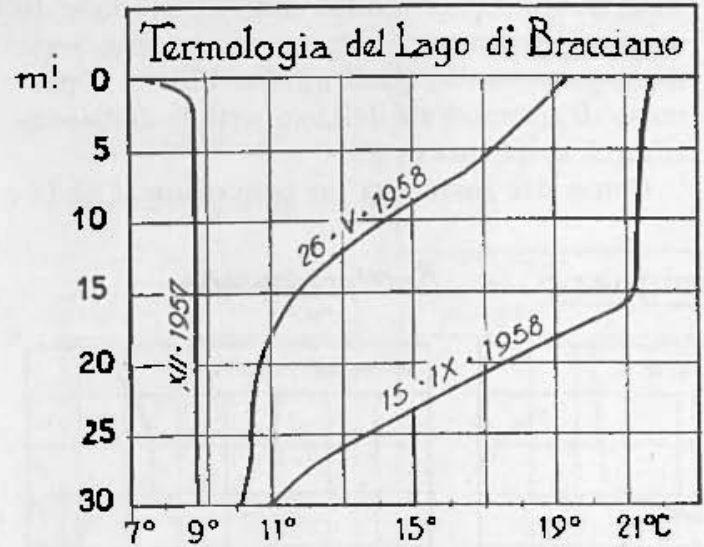

Fig. 12

Il campo di misura delle temperature è di circa $30^{\circ} \mathrm{C}$.

1 'C di variazione della temperatura, corrisponde a circa num 0,6 di deviazione sulla carta cerata.
Per quanto concerne l'apparecchio registratore, esso è corredato di due orologerie intercambiabili: una puó consentire duc avanzamenti diversi della carta, di $10 \mathrm{~mm} /$ ora o di $60 \mathrm{~mm} / 0 \mathrm{ra}$, con intervallo di registrazione di 1 minuto e carica di 8 giomi; l'altra consente pure due velocità di avanzamento, di $120 \mathrm{~mm} /$ ora e di $1200 \mathrm{~mm} /$ ora rispettivamente, con registrazione ogni 12 sec c carica di 28 ore.

Il tubo Bourdon puó essere tarato prina. e dopo ogni misura, mediante una bilancia. di pressione oppure con manometro di prova.

La lamina bimetallica puó essere tarata con un termografo.

La deterninazione dei valori assoluti del livello d'acqua viene ottennta calcolando lc correzioni per le variazioni termiche e barometriche.

3. - Con l'apparcchio brevemente descritto nel paragrafo precedente, mi sono proposto di compiere un certo numero di

\section{Lago di Bracciano. Trevignano. Batitermografia}
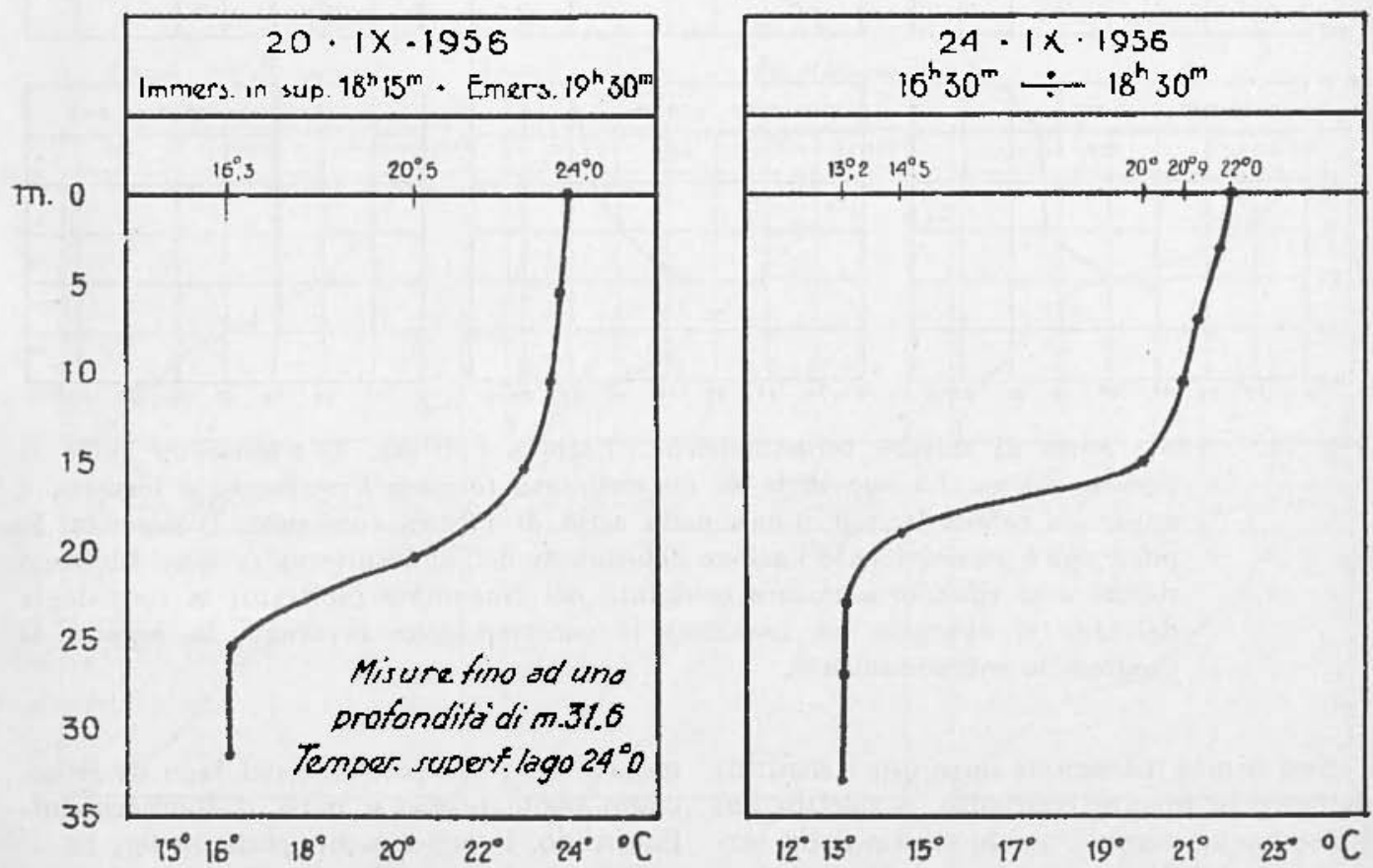

Fig. 13. - 1956: Prime esperienze batitermografiche nel lago di Bracciano: apparisce netto lo strato del salto termico a circa 16 metri di profondità. 
osservazioni termiche nelie acque del lago, in epoche diverse, allo scopo di rivelare se esisteva - il salto termico, necessario alla formazione della cosi detta superficie di discontinuità termica e, quindi, delle onde interne (sesse termiche). diagrammi (ho ritenuto superfluo, dato lo scopo di questa ricerca, unire lunghe serie di numeri), dalle quali appare chiaro il processo di formazione della superficie di discontinuità sospettata.

Come del resto era da prevedere, l'anda-

\section{Lago di Bracciano - Trevignano - Batitermografia}
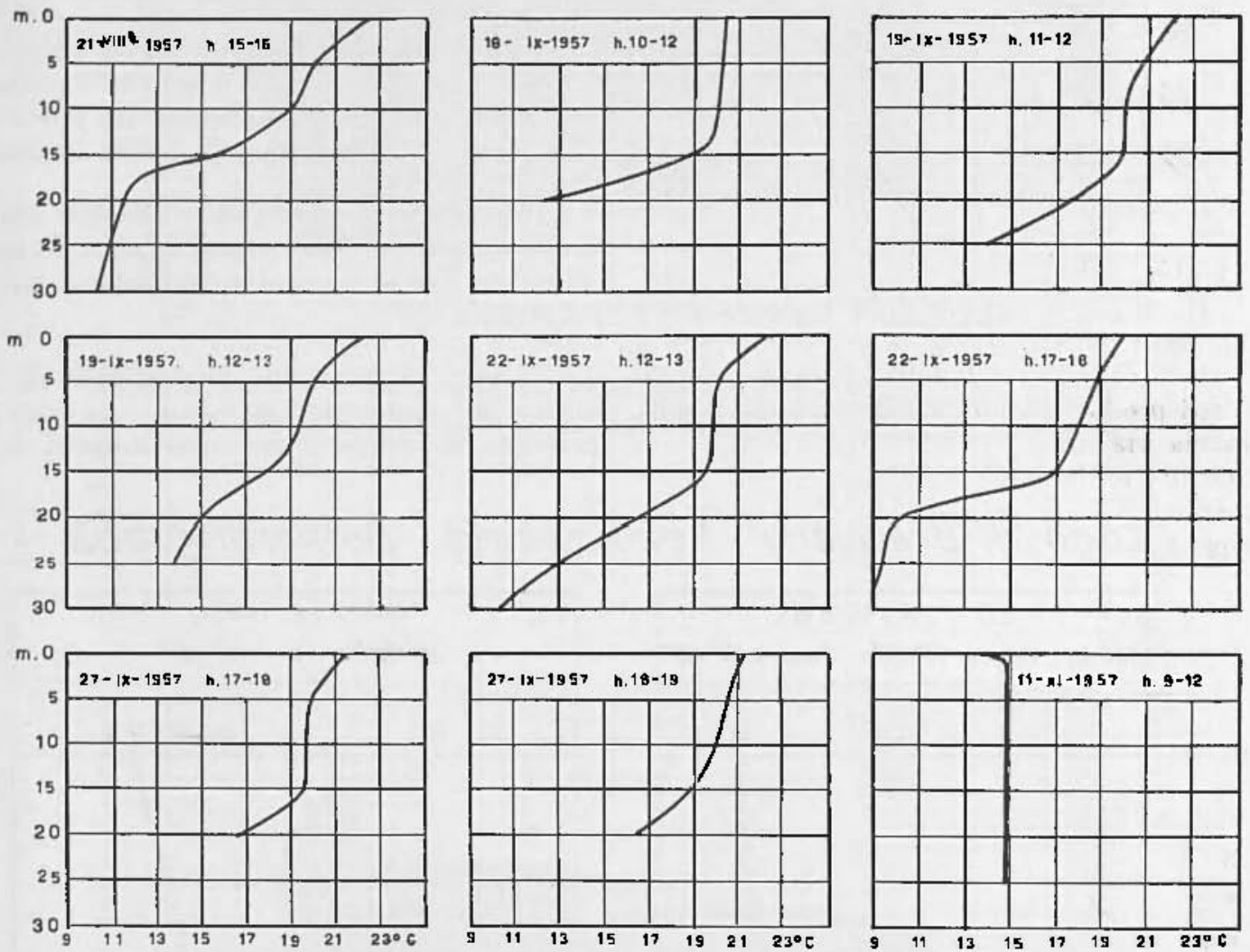

Fig. 14. - 1957: Serie di misure termometriche. Esclusa l'ultima, si riferiscono tutte al periodo estivo. La superficie di discontinnità termica è certamente formata $\theta$ apparisce chiara in più d'una delle serie di misure compiute. D'altronde, in più d'una è pure evidente l'azione deformante dell'onda interna in atto. L'ultimo riquadro si riferisce a misure compiute nel Novembre inoltrato; la termologia del lago si appresta ad assumere le caratteristiche invernali, in seguito aI progressivo raffreddamento.

Non è mia intenzione dare qui i risultati di tutte le misure compiute, a partire dal 1956 ; anche perchè, per lo studio della termologia del lago, mi riservo di compiere indagini più sistematiche, con mezzi più. idonci. Mi limito a riportare alcune serie di mento della temperatura nel lago di Bracciano segue presso a poco, dall'inverno all'autunno, lo schema riportato in fig. 12.

J) valori pressochè costanti lungo la verticale, dei mesi invernali, la temperatura, nella primavera, comincia a crescere negli 
Lago di Bracciano

Trevignono

Batitermografia
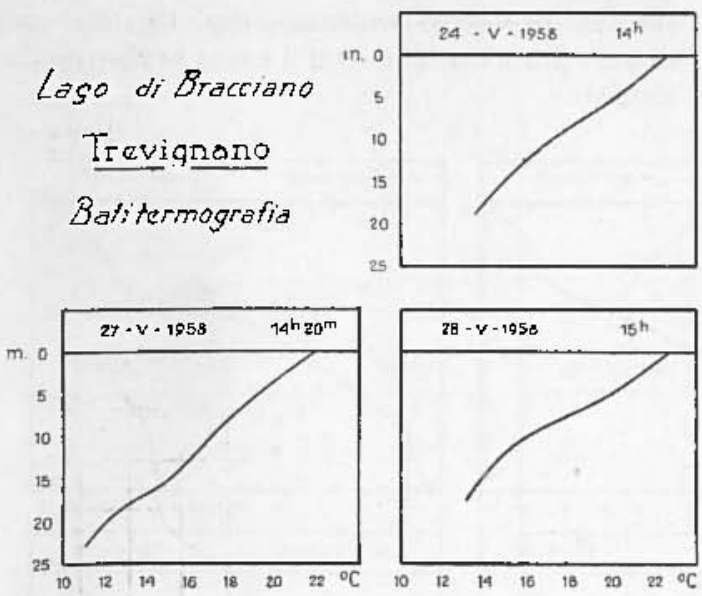
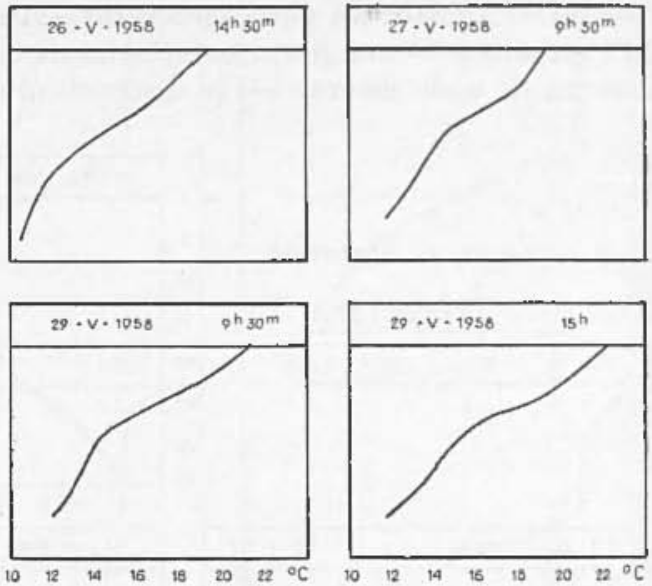

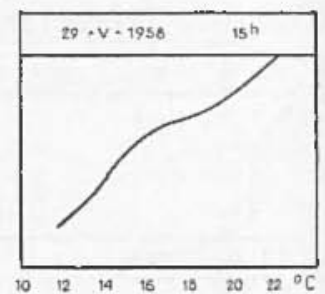

Fig. 15. - 1958; Serie di misure di fine Maggio. L'andamento della temperatura con la profon. dità sta rapidamente avvicinandosi al regime estivo.

strati prossimi alla superficie; l'aumento si fa via via più manifesto procedendo verso l'estate, e la temperatura assume un andamento deI tjpo di quello riprodotto nelle figg. 15,16 .
Alla fine di Luglio già si delinea, negli struti superficiali, la situazione termica da cui avrà origine lo strato di salto - e quindi la superficie di discontinuità. Quest'nltima già si presenta chiaramente nella seconda

\section{Lago di Bracciano. Trevignano. Batitermografia}
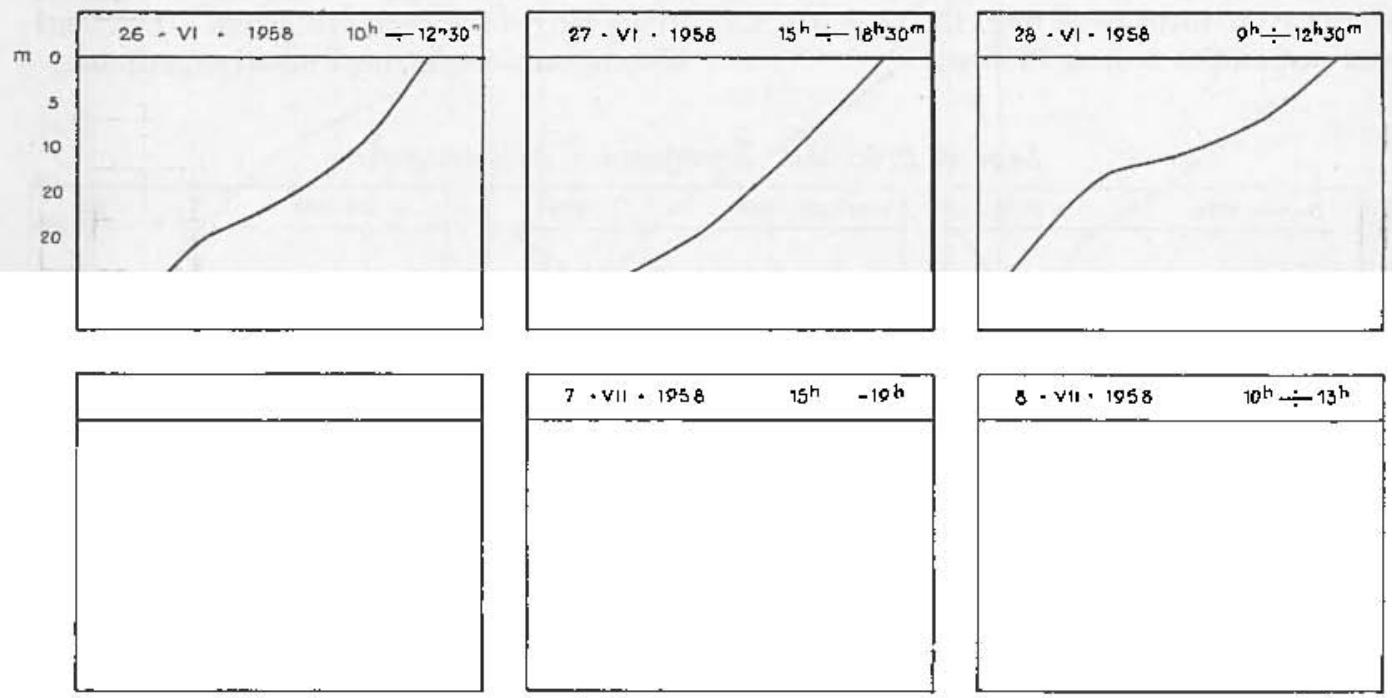

Fig. 16. - 1958: Serie di misure fine Giugno-prineipio Luglio. La zona del salto termjeo è già virtualmente formata, anche se ancora instabile e, comunque, alterata dalle onde interne. 
decade di Agosto, sia pure ancora instabile. In Settembre - nei giorni caratterizzati da assenza di onde interne - la discontinuità tinuità pressochè scompare (fig. 14). Ne vedremo più avanti il - del resto evidente motivo.
Lago di Bracciono

Trevignano

Batitermografia
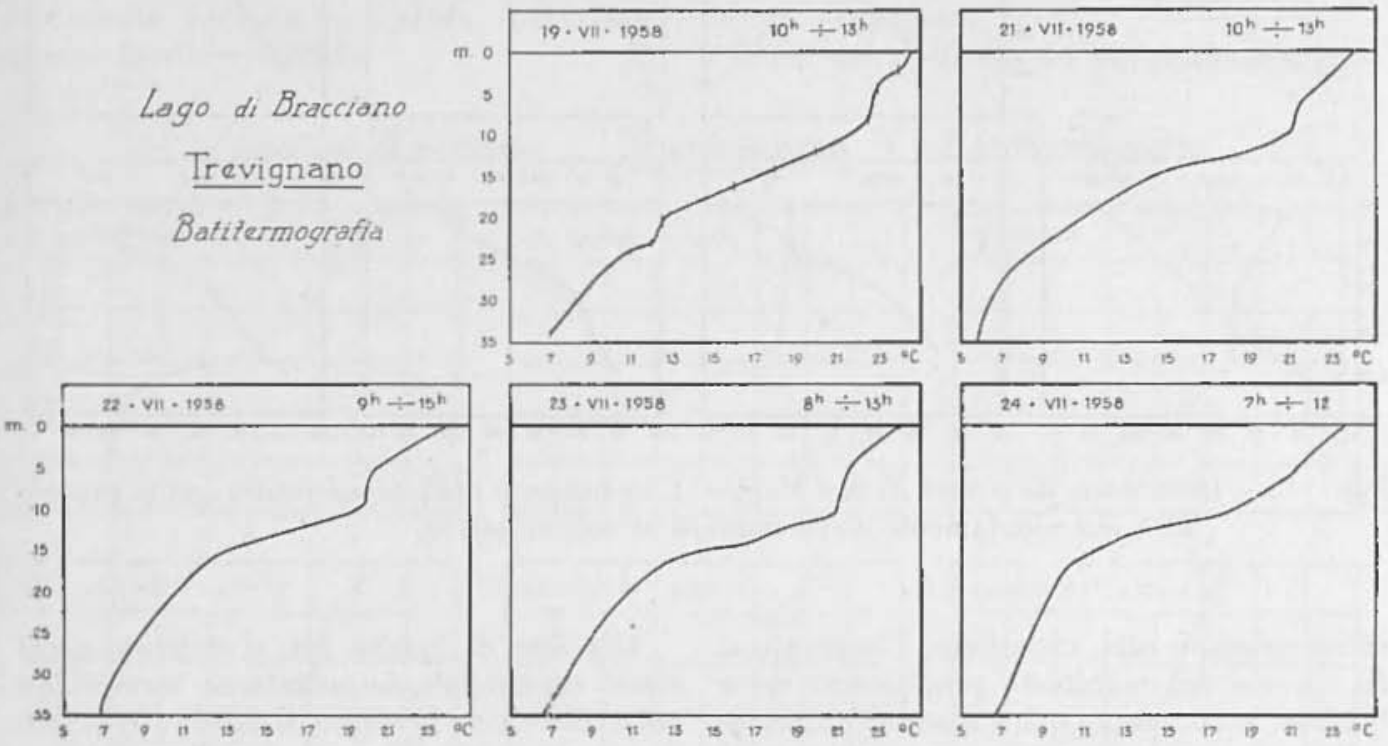

Fig. 17. - 1958: anche nella seconda metà di Luglio, la zona del saito termico, forse ancora instabile, è alterata dalle onde interne.

termica è in netto risalto, e si presenta nella forma riprodotta nelle figg. 13,14 e 19. La sua profondità de di $m 16$ circa.
Col sopraggiungere dell'autunno, il graduale raffreddamento dell'acqua - più sensibile, naturalmente, negli strati superficiali -

\section{Lago di Bracciano. Trevignano - Batitermografia}
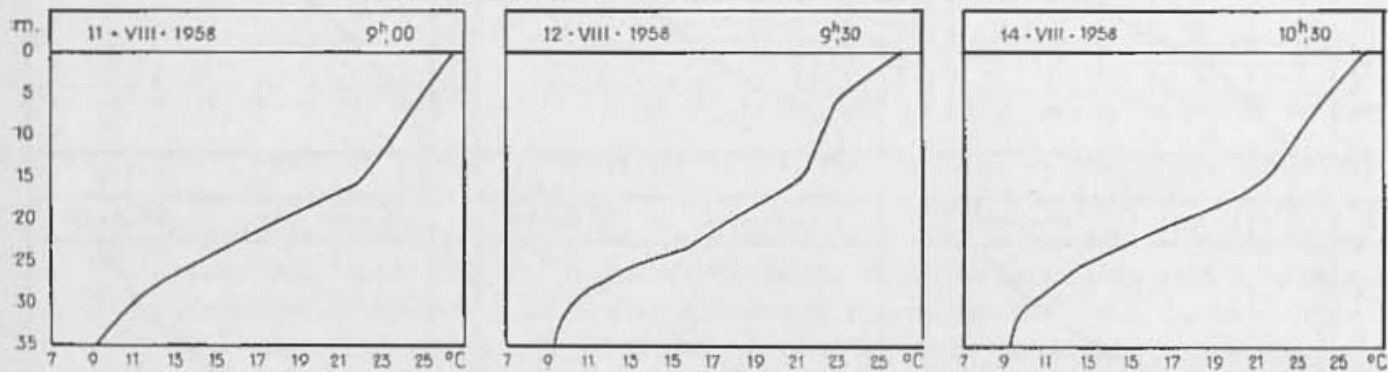

Fig. 18. - 1958: Nelia prima meta di Agosto, la superficie di discontinuità termica è nettamente delineata.

Nei giorni in eui si formano oscillazioni interne, allora la distribuzione termica degli strati superficiali viene più o meno profondamente alterata e la superficie di discon- finisce con l'uniformizzare la temperatura del lago, provocando la scomparsa della discontinuità termica e, quindi, delle condizioni necessarie per la formazione delle onde interne. 
Lago di Bracciano. Trevignano - Batitermografio
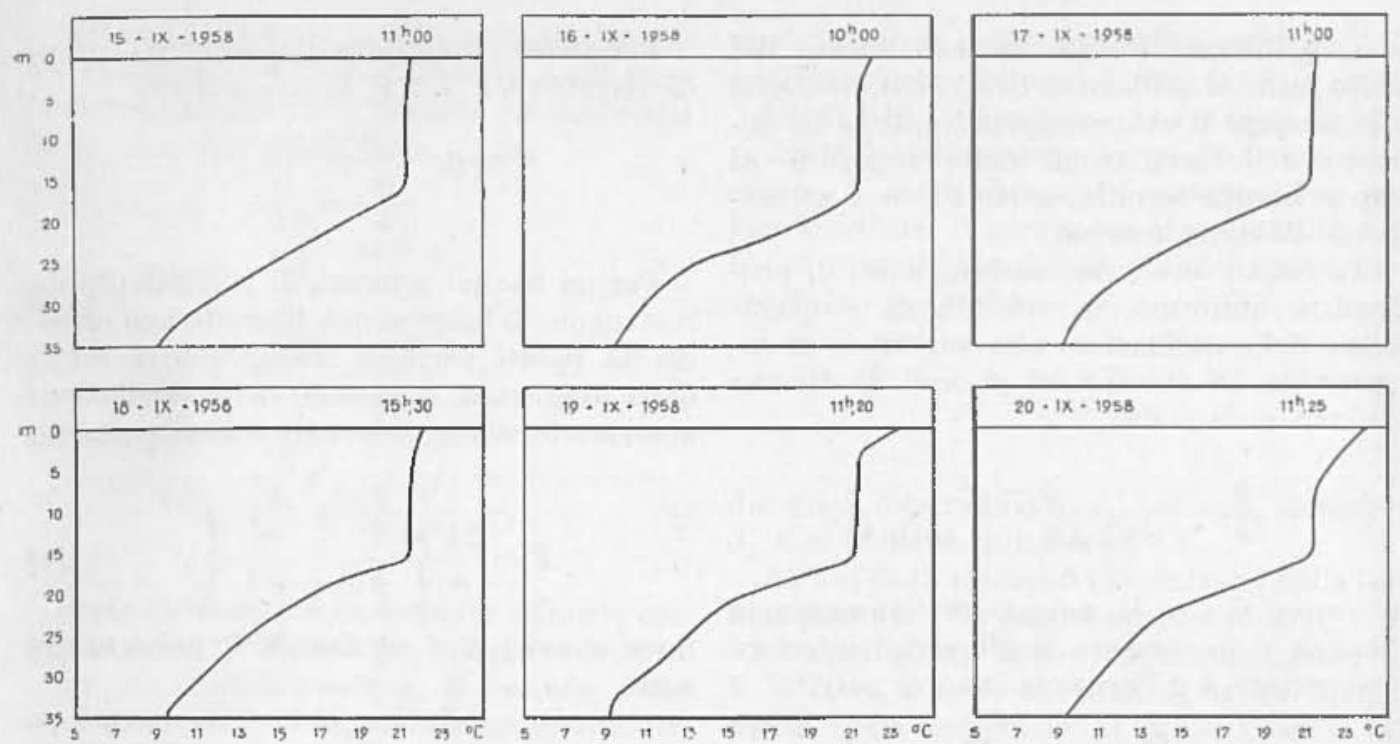

Figr. 19. - 1958: Dal 15 al 20 Settembre, la superficie di discontinuità termica, non perturbata da onde interne, si presenta nottissima alia profondità di $16 \mathrm{~m}$ circa.

Lago di Bracciano. Trevignano. Batitermografia

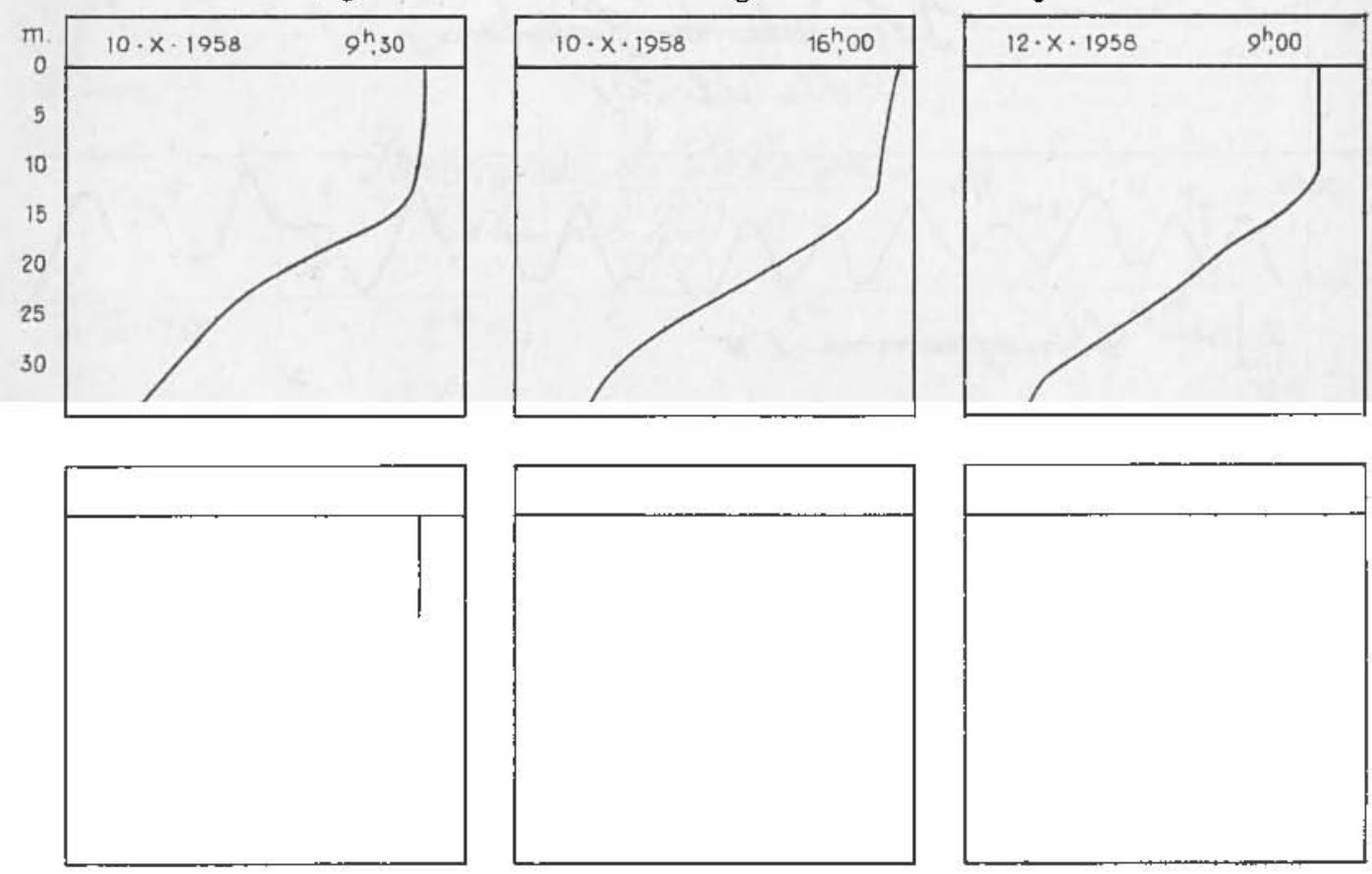

Fig. 20. - 1958: Dal 10 al 14 Ottobre la superficie di discontinuità è stata sorpresa nella sua fase di solievamento, sotto l'azione delle onde interne. 
4. - Provuta l'esistenza dello strato del salto termico nel lago di Bracciano, vediamo di calcolare il valore approssimativo del periodo delle oscillazioni della superficie di discontinuità termica, sotto l'azione perturbante di cause esterne.

In canali di sezione rettangolare, di profondità uniforme, la velocità di propagazione delle oscillazioni alla superficie di separazione di due liquidi pesanti di diversa densità è data da

$$
c^{2}=\frac{g}{k} \frac{\varrho-\varrho^{1}}{\varrho \operatorname{coth} k h+\varrho \operatorname{coth} k h^{\prime}},
$$

dove $\varrho$ e $h, \varrho$ e $h^{\prime}$ sono rispettivamente la densità e lo spessore degli strati inferiore e superiore, e $g$ l'uccelerazione di gravità.
Per onde di lunghezza d'onda $(2 \pi / k)$ grande rispetto a $h^{\prime}$ e a $h$, si può scrivere

$$
c^{\circ}-g \frac{\varrho-\varrho}{\frac{\varrho}{h}+\frac{\varrho^{\prime}}{h^{\prime}}} \text {. }
$$

Per un bacino a forma di parallelepipedo rettangolo di lunghezza $l$, limitato agli estremi da pareti verticali, ortogonali al senso della lunghezza, il periodo delle oscillazioni stazionarie, di cui puó essere sede, è pertanto

$$
T=\frac{2 l}{n}\left\{\frac{\frac{\varrho}{h}+\frac{\varrho^{\prime}}{h^{\prime}}}{g\left(\varrho-\varrho^{\prime}\right)}\right\}^{\top / 2},
$$

dove $n(=1,2, \ldots)$ denotia il numero dei nodi.

\section{Marea-Rapp. 1/100}

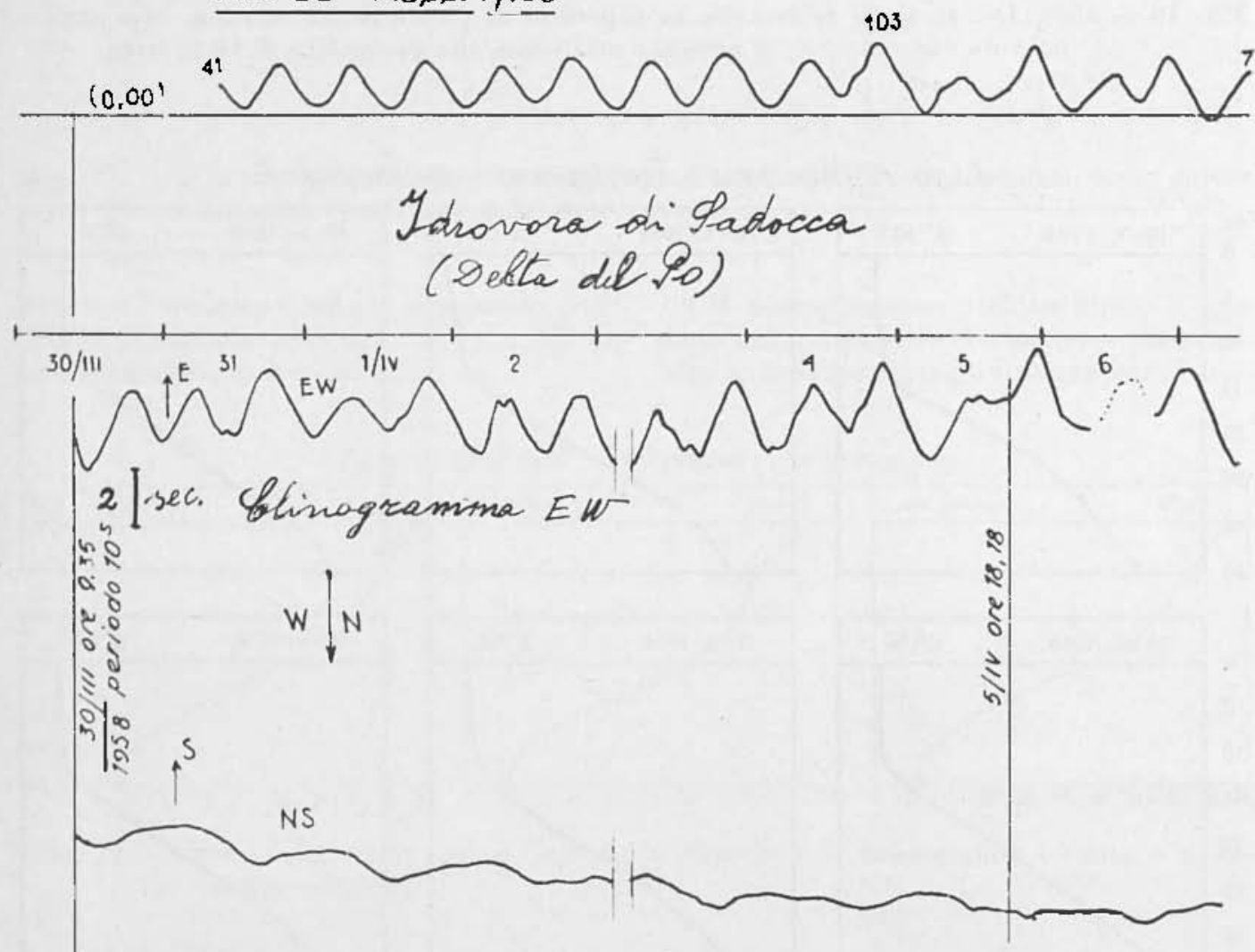

Fig. 21. - Marea solida (deviazione verticale apparente) registrata a Sarlocca dalla comp. EW del clinografo ivi funzionante, in corrispondenza della marea liquida sopra riportata nel rapporto $1 / 100$. 
Nel caso in esame, la differenza di densità fra gii strati è dovuta alla differenza di temperatura; inoltre $h^{\prime}$ è piccolo nei confronti di $h$, così che ponendo

si ha

$$
\frac{h^{\prime}}{h}=\varepsilon,
$$

avendo posto

$$
T=\frac{2 l}{n} \frac{1}{\sqrt{g h_{o}}},
$$

$$
h_{o}=\frac{h^{\prime}\left(\varrho-\varrho^{\prime}\right)}{\varrho+\varepsilon \varrho} .
$$

Sotto la forma [2], la formula coincide con quella detta di Merian per le sesse ordinaric.

In via approssimativa, il periodo delle sesse interne di un lago di forma qualunque puó quindi essere determinato con la [2], dove in luogo della profondità effettiva va considerata la profondità ridotta [3].

Per il lago di Bracciano, la cui forma - in prima upprossimazione - può ritenersi circolare, $h^{\prime}$ è sensibilmente costante. Per un lago circolare di raggio $r$ e di profondità $h_{s}$, e nel caso di lunghezze d'onda grandi rispetto ad $h_{a}$, si ha

$$
T=\frac{2 \pi \cdot r}{x_{i} / g h_{o}},
$$

dove $x_{i}$ è una radice di $J_{1}^{\prime}(x)=0$, essendo $J_{1}$ una funzione di Bessel.

Alcuni degli eicmenti che entrano nella [4] non possono the essere approssimativi. Ad ogni modo, con buona approssimazione, ì $h=15 \mathrm{~m}, h=65 \mathrm{~m}$. Inoltre, in corrispondenza delia superficie di discontinuità, si può assumere $r=3500 \mathrm{~m}$. I valori medi

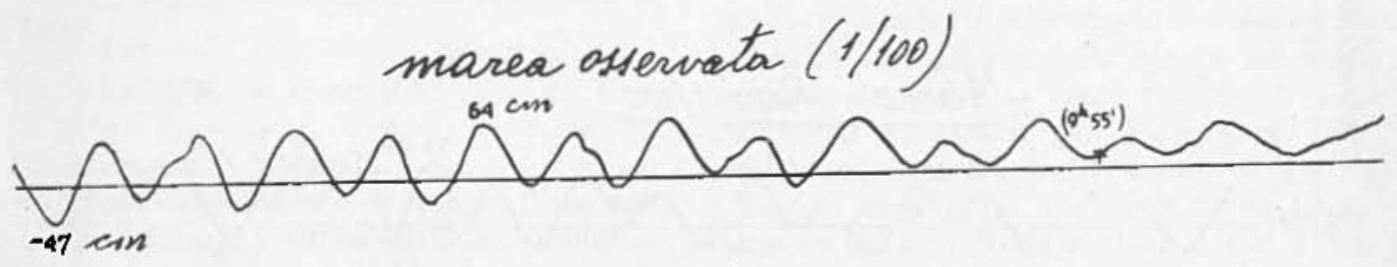

$$
\frac{\text { Ydrovora di Sadocca }}{\text { (Deta del }}
$$

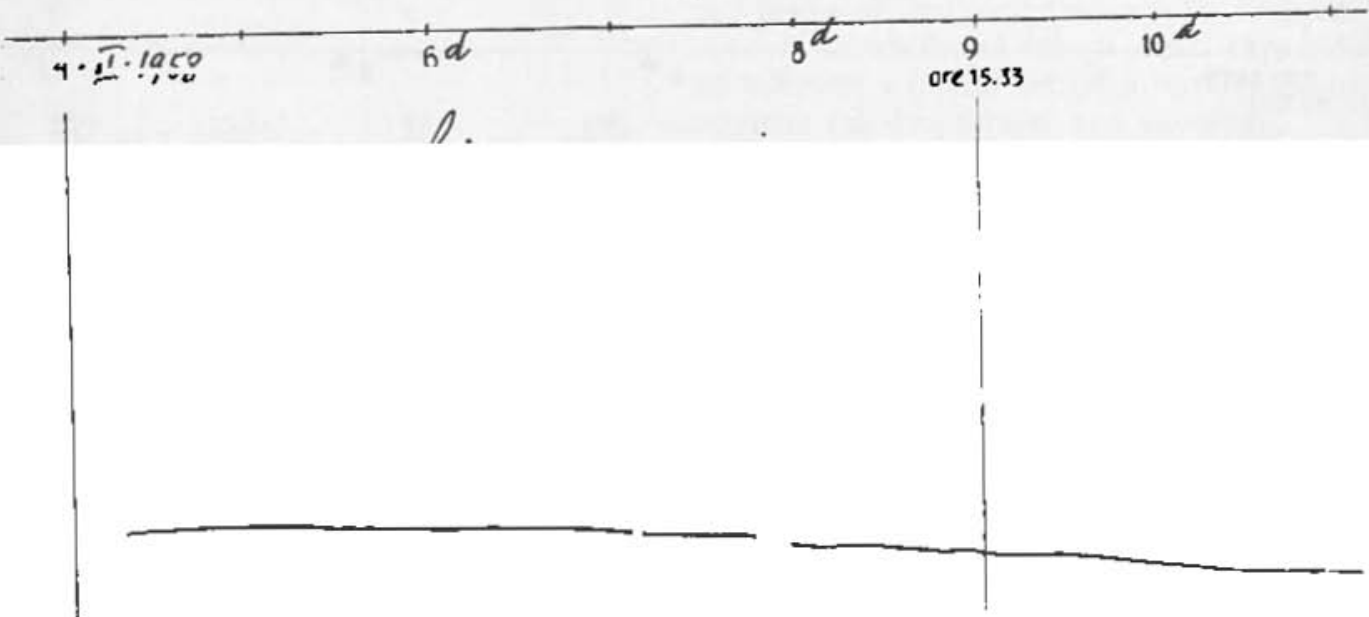

Fig. 22. 
delle temperature osservate nei due strati, superiore ed inferiore, furono di $24^{\circ}$ e $9^{\circ}$ rispettivamente, ai quali corrispondono densità dell'ordine di $\varrho=0.997296$ e $\varrho=$ 0.999781 .

Questi dati permettono di ottenere (fatto $\left.g=9,81 \mathrm{~m} / \mathrm{sec}^{2}\right) \mid g h_{o}=0.5603$.

Si sa che le radici di $J_{2}^{\prime}(x)=0$ sono, nell'ordine,

\section{$1.841 ; 5,33 ; 8,54$;}

Al primo di questi valori, corrisponde tenendo conto della [4] — per l'onda interna. uninodale il valore di

$$
T_{0}=6^{\mathrm{n}}, 0 \text {. }
$$

In questi calcoli, naturalmente, quello che conta è l'ordine di grandezza dei valori per- seguiti. Nel nostro caso, tale valore non potrebbe essere più significativo.

5. - La teoria prevede dunque un periodo di $6^{\text {h }}$ circa per l'onda interna uninodale del lago di Bracciano, quale conseguenza della sua particolare termologia estiva - autunnale.

Gli esempi riportati nelle figg. 1-8, dedotti dalla riduzione delle registrazioni delle sesse ordinarie del lago, erano già di per sè significativi e costituivano un'ottima conferma dei valori ottenuti per via analitica.

Ho cercato peró di ottenere la registrazione delle onde interne, il più possibile scevre dalle perturbazioni derivanti dalle oscillazioni libere ordinarie, a più breve periodo. Ciò comportava l'opportuno fil-

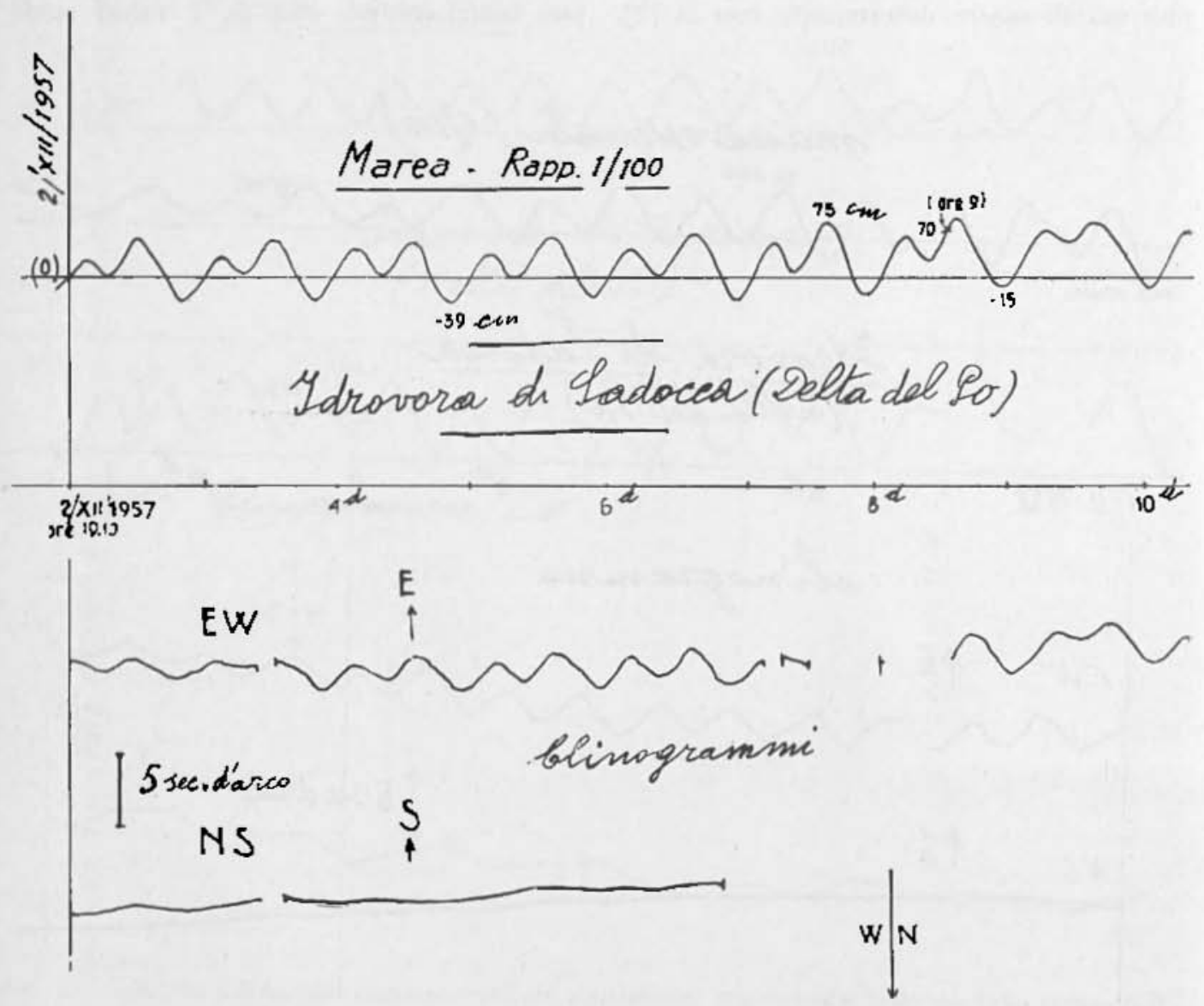

Fig. 23. 
traggio dei limnografi che permettesse di eliminare le oscillazioni a breve periodo.

Si è ottenuto lo scopo valendoci dei principi stabiliti nel precedente lavoro, trattando della parte strumentale $\left(^{2}\right)$.

L'applicazione di un filtro ai limnografi provoca ritardo $(\tau)$ nella registrazione $\mathrm{e}$ smorzamento nell'ampiezza nel rapporto $\cos \omega \tau: 1$, essendo $\omega=\frac{2 \pi}{T} \quad(T$, periodo dell'oscillazione.

Si ha $\left({ }^{2}\right)$

$$
\frac{\tau}{T}=\frac{1}{2 \pi} \operatorname{arctg} \frac{\omega}{k},
$$

essendo

$$
k=2890 \frac{b^{1}}{l a^{2}},
$$

dove $l$ ì la lunghezza del tubo adduttore, di diametro $b$, nel pozzetto cilindrico di diametro $a$.

Per la registrazione delle onde interne si è fatto

$$
l=\mathrm{cm} 400, \quad b=\mathrm{cm} 0,3, \quad a-\mathrm{cm} 6 .
$$

Consegue $k=0,001626$.

Ho calcolato l'effetto di questo filtro sulle sesse ordinarie e sull'oscillazione uninodale delle onde interne. I risultati del calcolo sono contenuti nella seguente tabella:

\begin{tabular}{cccc}
\multicolumn{4}{c}{ per $k=0,001626$} \\
sec & $\tau / T$ & $\begin{array}{c}\tau \\
\text { sec }\end{array}$ & $\cos \omega \tau$ \\
492 & $\cdot 2304$ & 113 & $\cdot 126$ \\
330 & $\cdot 2364$ & 78 & $\cdot 085$ \\
240 & $\cdot 2401$ & 58 & $\cdot 062$ \\
21.600 & $\cdot 0282$ & 609 & $\cdot 984$
\end{tabular}

Appare chiara l'azione del filtro sulle oscillazioni ordinaric: esse vengono praticamente eliminate dalla registrazione. Infatti, già l'uninodale $(492 \mathrm{sec})$ è ridotta di $12 / 100$ circa; e quindi - data la sua piccola ampiezza a non più di un paio di millimetri nelle sue più vistose manifestazioni. Scompaiono senz'altro la binodale $\left(330^{3}\right)$ e l'uninodale simmetrica $\left(240^{5}\right)$.
Al contrario, l'onda interna uninodale $\left(21600^{\mathrm{s}}=6^{\mathrm{h}}\right.$ circa) viene riprodotta pressochè in vera grandezza $(98 / 100)$, con un ritardo di circa $10^{\text {th }}$ (unica sensibile azione (del filtro).

II limnografo cosi modificato è rimasto in funzione per lunghi periodi, durante gli ultimi due anni. Le registrazioni avute non potevano essere più significative.

Nei mesi da Novembre a Giugno, si ebbero - negli intervalli di funzionamento dell'apparecchio - registrazioni di nessun rilievo, comunque non attribuibili a moti interni, di origine termica.

Nelle settimane di funzionamento delle stagioni estiva-autnnnale si sono avute, invece, registrazioni di grandissimo interesse: a periodi di quiete, si sono alternati intervalli caratterizzati da oscillazioni veramente cospicue. Si vedano gli esempi riportati nelle Tavole da I a IV.

Stupisce la persistenza e l'ampiezza delle oscillazioni, aventi un periodo di 6 ore circa.

Cол la formazione dello strato del salto termico, si creano - come si è visto - le condizioni necessarie per l'insorgere delle oscillazioni interne. Sulla causa di queste ultime ci riserviamo di ritornare più partitamente nel seguito della ricerca. Fin d'ora, possiamo osservare che una perturbazione della superficie esterna - quale può essere quella legata a vento improvviso 0 al transito di disturbi microbarici - è sufficiente per provocare un'oscillazione della "superficie " di discontinuita fra gli strati superiore ed inferiore a quello del salto termico, come avviene fra due liquidi nоп miscibili.

Questo peró non spiega la persistenza delle onde interne, che durano settimane, e la loro ampiczza veramente eccezionale.

Va ricordato, a questo proposito, che l'am. piezza delle onde interne alla superficie esterna del mezzo è proporzionale alla differenza $\varrho-\varrho^{\prime}$, sempre piccolissima. Wedderburn escludeva che esse potessero impressionare la superficie, tanto da negare che le lievi oscillazioni rilevate da Halbfasse nel Madusee fossero reali onde interne. In realtà, qualche sporadica registrazione di onde interne alla superficie esterna si è avuta in qualche lago giapponese, mentre ampie si manifestano nel lago di Garda (1). 
Nel caso del lago di Bracciano, ritengo che la persistenza e l'ampiezza delle onde interne comporti l'esistenza di fenomeni di risonanza con la cansa perturbante . E qui - dato il periodo proprio delle onde interne - viene spontaneo di pensare all'azione indiretta della maren.

L'attrazione diretta luni-solare, naturalmente, non può avere efforcia alcuna: solo nei massimi laghi della Terra, infatti, essa. può destare movimenti piccolissimi, dell'ordine del millimetro.

Ja marea liquida può agire per via indiretta. La marea luni-solare semi-rliurna è pressochè sempre presente nel Tirreno: la. sua armonica superiore, avrebbe il periodo dell'onda interna del lago di Bracciano. Del resto, non è da escludere che possano verificarsi maree quarto-diurne, sia pure di piccola ampiezza, aventi appunto il periodo di $6^{\text {h }}$.

L'xzione sull'onda interna del lago di Bracciano può manifestarsi solo attraverso la crosta terrestre, sotto forma di marea solida.

Che la marea liquida possa trasformarsi in maren solida di ugual periodo è cosa nota. Nelle figg. 21, 22, 23, vengono riportati tratti di marea solida, che viene sistematicamente registrata presso l'idrovora di Sadocea. (Dclta del Po) in corrispondenza di quella liquida, da un fotoclinografo ivi funzionante dal Dicembre 1957.

E chiaro che l'onda solida si smorza rapidamente con la distanza; è pur vero però che, nei fenomeni di risonanza, l'ampiezza. (intensità) della causa perturbante non ha influenza determinantc, che è invece essenzialmente legata all'approssimazione dei periodi in giuoco ed è tanto maggiore quanto più il periodo della cansa perturbante si approssima a quello del sistema oscillante.

\section{RIASSUNTO}

Sono proseguite le indagini, iniziate gli anni scorsi, sul lago di Bracciano, con particolare riguardo alla termologia del lago stesso. Attraverso le quattro stagioni, sono state compiute numerose serie di misure di temperatura, a diverse profondità, con un batitermografo fatto espressamente costruire. Le misure ottenute hanno già consentito di delineare l'andamento della temperatura delle aeque del lago durante l'anno e a dare conferma definitiva del formarsi, durante $i$ mesi da Luglio a Novembre, dello strato del salto termieo. La base di detto strato si comporta come superficie di discontinuita, che praticamente divide il lago, nei mesi accennati, in due parti non miscibili, di diversa densità. Ciò crea le condizioni fisiche necessarie e sufficienti all'insorgere delle cosi dette "onde interne" (sesse termiche), sotto l'azione di cause perturbanti esterne: la superficie di discontinuità diviene sede di moti stazionari, $i$ cui periodi sono legati alle oaratteristiche morfologiche e di densità del lago, di ampiezze molto spesso notevoli. Poichè tali onde si smorzano rapidissimamente verso la superficie esterna, era opinione diffusa che esse non potessero venir registrate in superficie, come onde idriche, se non sporadicamente. Nel lago di Bracoiano, invece, vengono registrate in superficie lunghissime serie di oseillazioni, aventi un periodo proprio di 6 ore oirca. La teoria ha provato che tali onde sono le fondamentali (uninodali) delle onde interne. Il fatto ohe esse vengano registrate, con inusitata ampiezza, durante intere settimane pù̀ forse essere attribuito ad un fenomeno di risonanza, quale potrebbe essere determinato da maree terrestri, conseguenti a maree quarto-diume, o armoniche di semidiurne del vicino Tirreno.

\section{ABSTRACT}

The study of the lake of Bracciano and especially of its thermology, undertaken a few years ago, has been continued. The temperature of its water has been taken frequently at various depths utilizing a specially designed and built batithermograph.

The data thus colleoted have already permitted to outline the trends of the temperature of the water of the lake during the year and to confirm the fact that, during the period from 
July to November, a thermic-jump layer materializes in it. The base of this layer behaves like a discontinuity surface which practically divides the lake into two parts endowed with different density, which do not mix together. This ereates physical conditions which are necessary and sufficient to create the socalled "internal waves". Owing to the effect of external perturbing causes, the discontinuity surfaee becomes the seat of stationary movements whose periods are linked with morphologic features and density of the lake and whose amplitude is often considerable. As such waves extinguish very rapidly towards the external surface, an opinion had spread that they could not be recorded at the surface as watery waves except in sporadic cases. In the lake of Bracciano, instead, very long series of aseillations with their own period of 6 hours have been recorded at the surface. A theory has proved that such waves are the fundamentals (uninodals) of internal waves.
The fact that they are registered, with unusual amplitudes, during whole weeks, can be, probably, attributed to a resonance phenomena which could be caused by earth tides deriving from quarter-day tides or half-day harmonics of the nearby Tyrhenian Sea.

\section{BIBILIOGRAFIA}

(1) Calor P., Sui possibili modi di oseillazione libera del lago di Bracciano, "Ann. di Geofisica ", III, 1 (1950).

(2) Calor P. e Peronaci F., Oseillazioni libere e forzate del lago di. Bracciano, "Arch. di Oceanogr. e Limm. n, Venezia, 1958.

(') CaLor P., Oscillazioni libere del lago di Bracciano; loro caratteristiche, loro energia, "Amm. di Geof. ", XI, 1 (1958).

(4) - Notevoli onde interne (sesse termiche), nel layo di Garda, "Ricerca scientifica ", $A$. XVII, l (1947). 


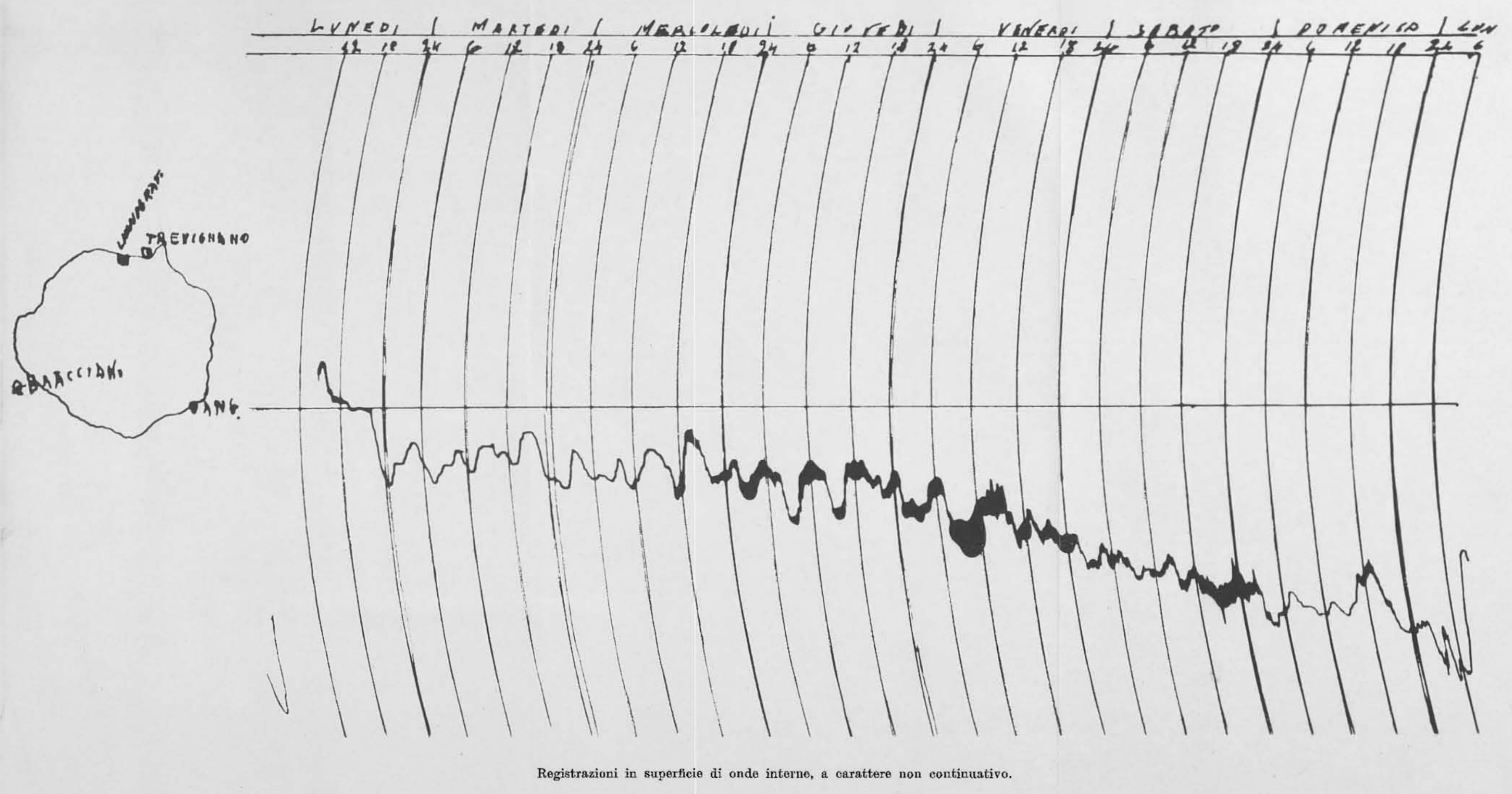




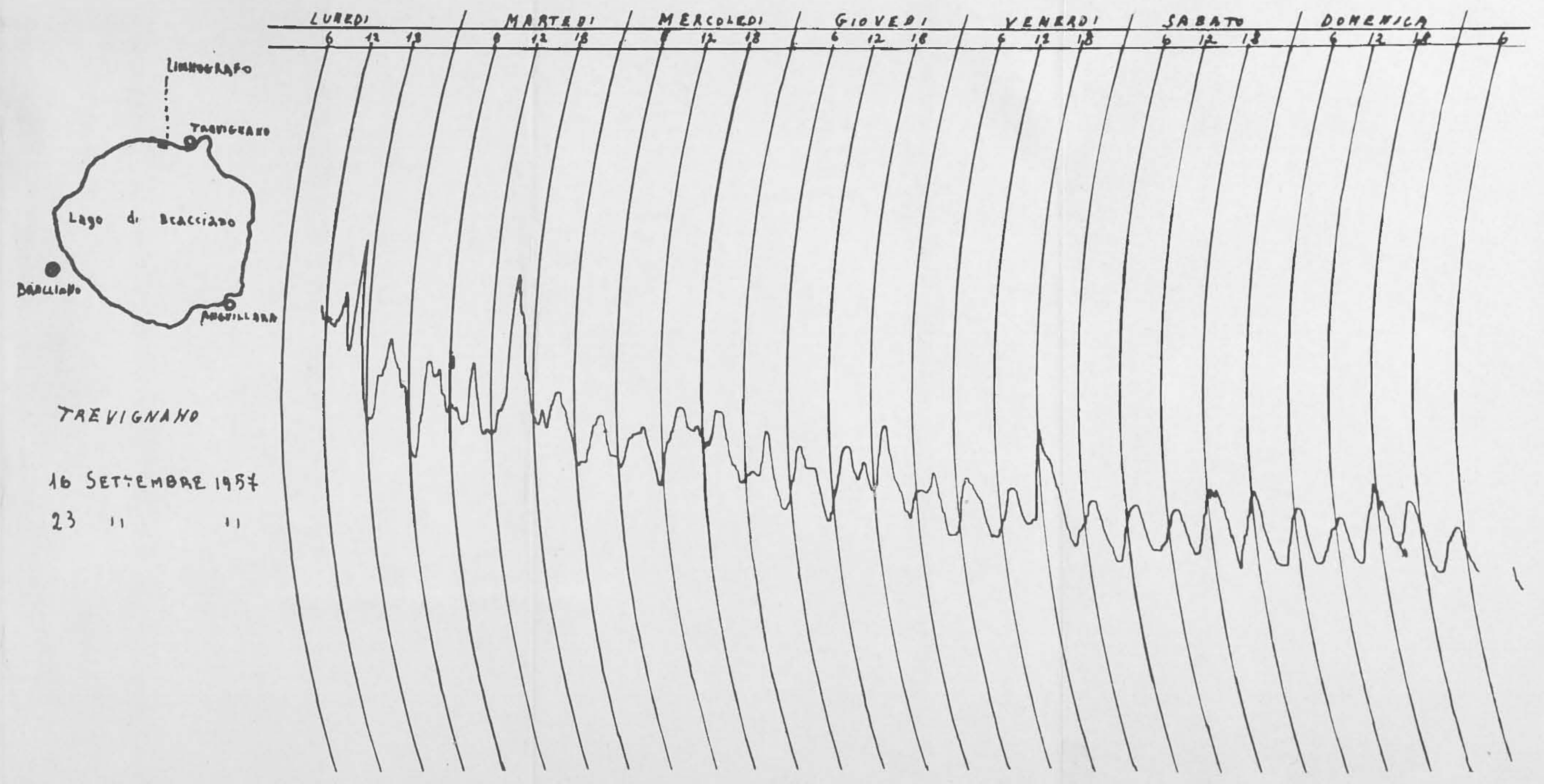




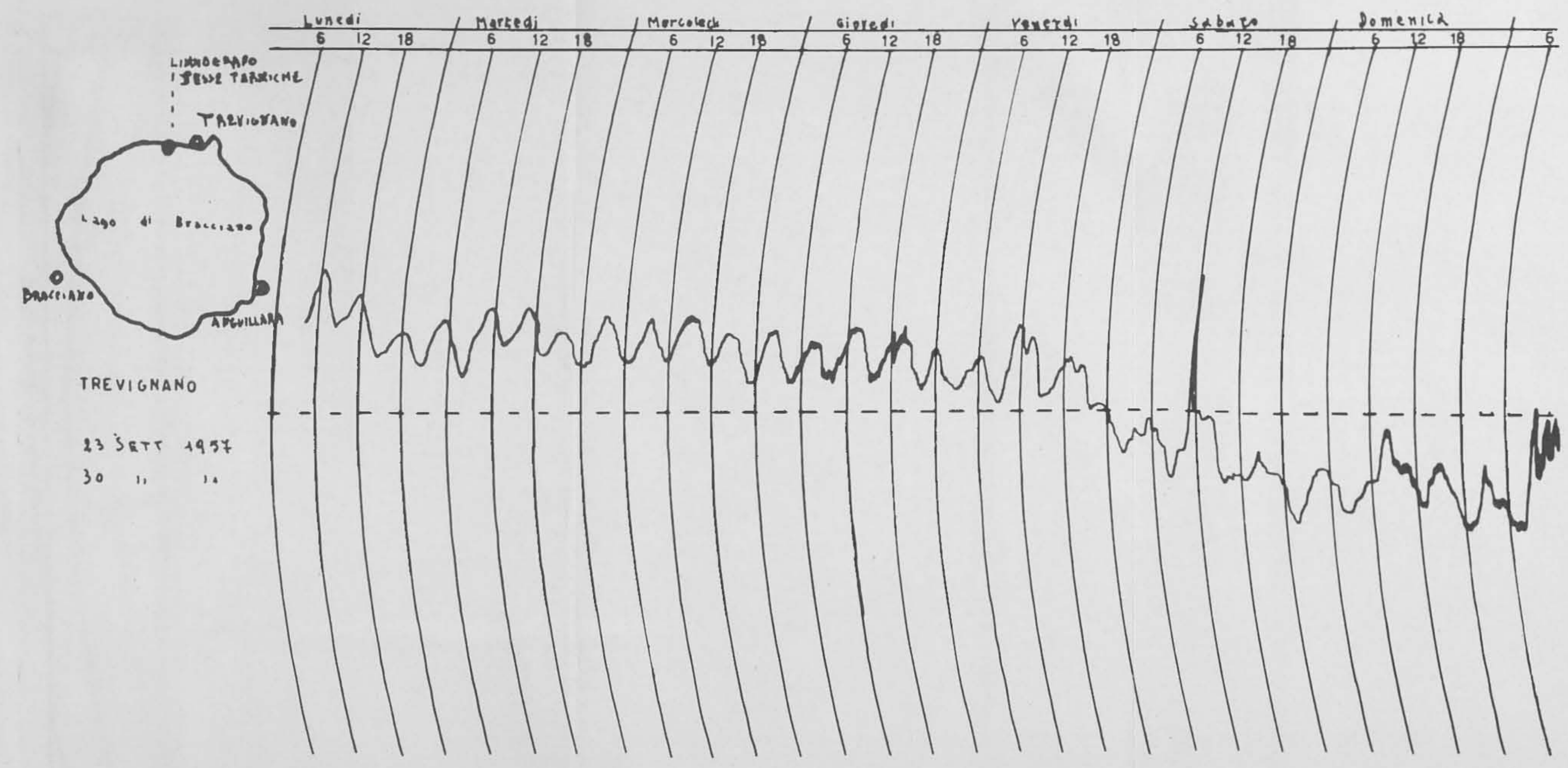




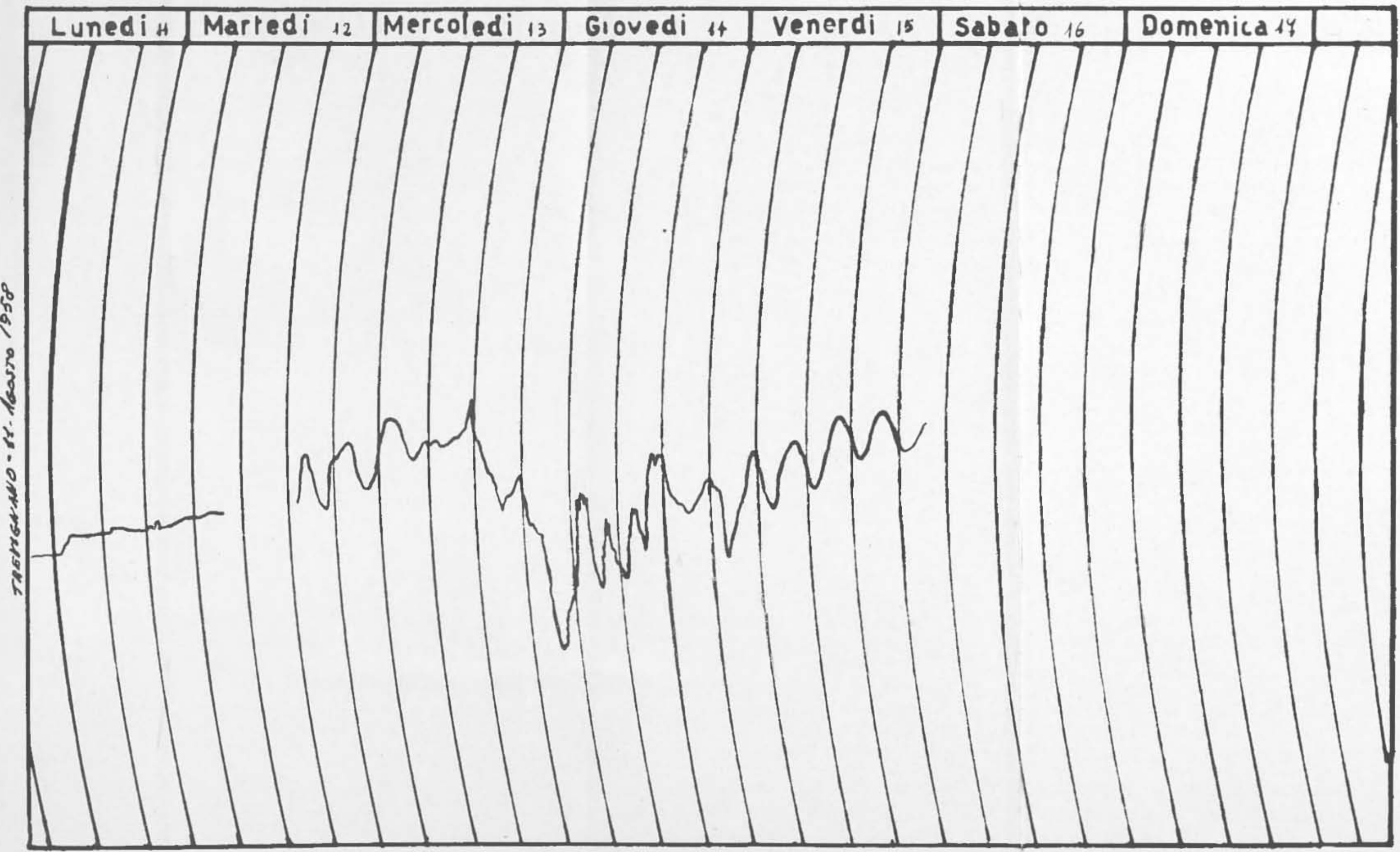

Dopo un periodo di relativa calma, la smperficie interna di discontinuita termica (strato del salto termico) si rimette in oscillazione: inizialmente, oltre ad onde uninodali, insorgono anche onde binodali; poi la serie uninodale continua quasi pura. 


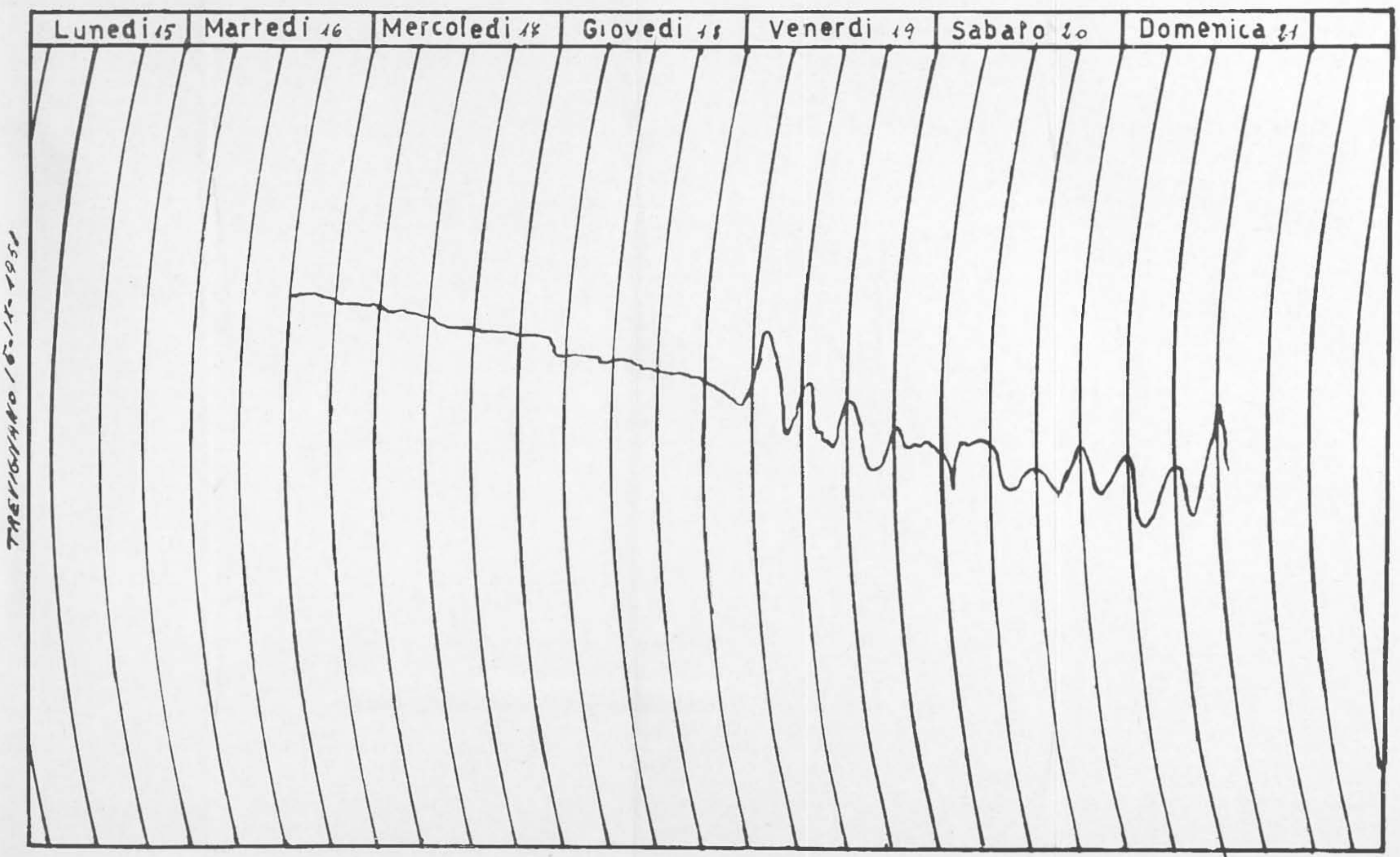




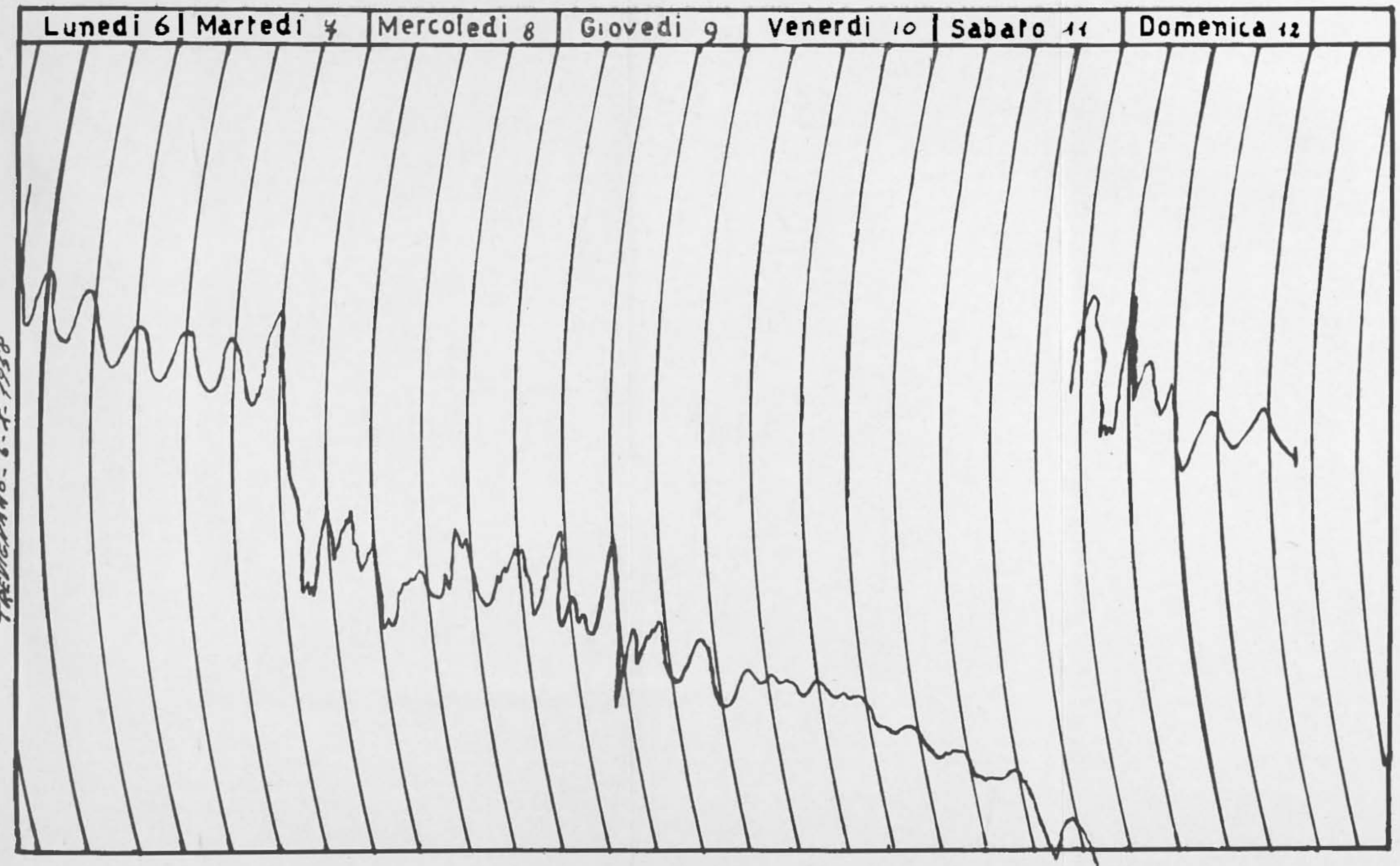

Il lago è stato sorpreso in una sequenza di ampie oscillazioni interne, regolarissime (6h di periodo ca.); seguono perturbazioni di altro periodo, mentro il livello del lago è in forte diminuzione (Registrazioni limnografiche superfieiali). 